\title{
Commuting Graphs of Finite Groups
}

\author{
Timothy Jon Woodcock \\ Belchertown, Massachusetts
}

Master of Science, University of Virginia, 2003
Bachelor of Science, Stonehill College, 1993

A Dissertation presented to the Graduate Faculty of the University of Virginia in Candidacy for the Degree of Doctor of Philosophy

Department of Mathematics

University of Virginia

December, 2010

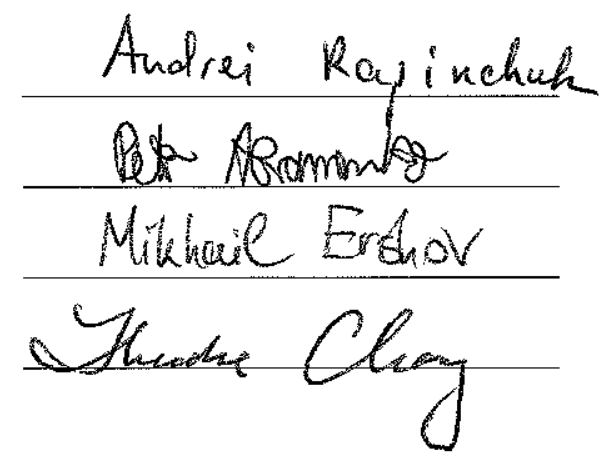




\begin{abstract}
The commuting graph of a finite group is defined to have the nontrivial elements of the group as its vertices, and an edge joining each commuting pair of elements. We explore the structure of the commuting graph for a variety of groups. In particular, the diameter of the commuting graph of the symmetric group $S_{n}$ is precisely described, based on the nature of $n$ and $n-1$. Furthermore the connected components of this graph are completely classified. We continue by establishing upper bounds on the diameter of the commuting graph for a certain class of solvable groups. Finally, we provide a structure theorem for groups of order $p^{a} q^{b}$ that consist strictly of $p$ - and q-elements, including a description of the commuting graph of such a group.
\end{abstract}




\section{Contents}

1 Introduction 1

1.1 Definitions . . . . . . . . . . . . . . . . . . 1

1.2 History . . . . . . . . . . . . . . . . . . 2

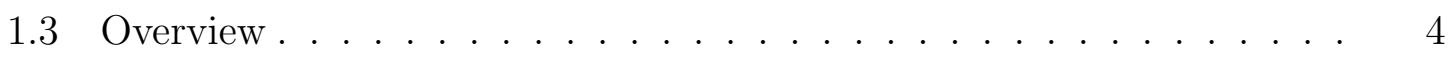

2 Symmetric Groups $\quad 6$

2.1 Main result ....................... 6

2.2 Commuting elements of $S_{n} \ldots \ldots \ldots \ldots \ldots$

2.3 The commuting graph $\Delta_{R_{n}} \ldots \ldots \ldots \ldots \ldots$

2.4 The commuting graph $\Delta_{R_{n} \cup C_{n}^{n}}$ for even $n \ldots \ldots$. . . . . . . . 16

2.5 An upper bound on the diameter of $\Delta_{S_{n}^{*}}$ for composite $n$ and $n-1$. 26

2.6 The existence of elements at distance 5 in $\Delta_{S_{n}^{*}} \ldots \ldots \ldots \ldots$

2.7 Proof of main result f . . . . . . . . . . . . . . . . . . 39

3 A Diameter Bounding Result and Certain Solvable Groups 41

3.1 Summary . . . . . . . . . . . . . . . . . . . . 41 


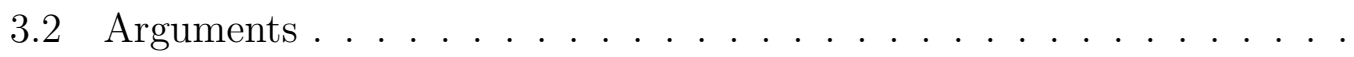

4 Groups of Order $p^{a} q^{b}$

4.1 Main results . . . . . . . . . . . . . . . . . . . . . . 49

4.2 The commuting graph $\Delta_{G^{*}}$ if $M(G)$ is nonempty . . . . . . . . 51

4.3 The structure of $G$ and $\Delta_{G^{*}}$ if $M(G)$ is empty . . . . . . . . . . 55

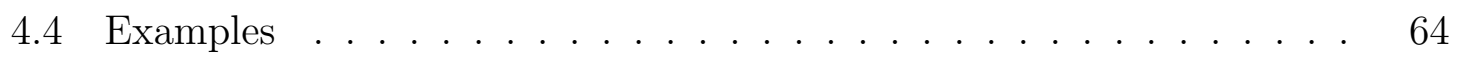

4.5 A condition for connectivity of $\Delta_{G^{*}} \ldots \ldots \ldots \ldots$. . . . . . 67 
For my wife, Suzanne,

my children,

and my parents. 


\section{Chapter 1}

\section{Introduction}

\subsection{Definitions}

Given a finite group $G$, we let $G^{*}$ denote the set of all nontrivial elements of $G$. For a nonempty subset $X$ of $G^{*}$, let $E_{X}$ denote the collection of all 2-element subsets $\{g, h\}$ of $X$ such that $g$ and $h$ commute, under the product of $G$. We define the commuting graph of $X$, denoted by $\Delta_{X}$, to be the ordered pair $\left(X, E_{X}\right)$. We remark that as we make use of this notation in our work of the following chapters, the group $G$ will be understood from context.

A sequence of elements $\left(x_{0}, x_{1}, \ldots, x_{i}\right)$ over $X$ is called a commuting path in $\Delta_{X}$, or a commuting path from $x_{0}$ to $x_{i}$, if for $j<i,\left\{x_{j}, x_{j+1}\right\} \in E_{X}$ or $x_{j}=x_{j+1}$. The length of a commuting path $\left(x_{0}, x_{1}, \ldots, x_{i}\right)$ is defined to be equal to the nonnegative integer $i$, which is one less than the number of terms in the path.

For an arbitrary pair of elements $g, h \in X$, possibly alike, we define the distance between $g$ and $h$ in $\Delta_{X}$ to be the minimum length of a commuting path from $g$ to $h$, provided that one exists. We denote this distance by $d_{\Delta_{X}}(g, h)$. If there is no 
commuting path from $g$ to $h$, we let $d_{\Delta_{X}}(g, h)=\infty$, and take the convention that $i<\infty$ for all nonnegative integers $i$. We note that $d_{\Delta_{X}}(g, h)=d_{\Delta_{X}}(h, g)$ in general, because $\left(g, x_{1}, \ldots, x_{i-1}, h\right)$ is a commuting path in $\Delta_{X}$ if and only if $\left(h, x_{i-1}, \ldots, x_{1}, g\right)$ is as such.

If $d_{\Delta_{X}}(g, h)<\infty$ for all $g, h \in X$, then we say that $\Delta_{X}$ is connected. Otherwise, $\Delta_{X}$ is said to be disconnected. If $Y$ is a maximal subset of $X$ such that $\Delta_{Y}$ is connected, then $\Delta_{Y}$ is called a connected component, or just a component, of $\Delta_{X}$. We define the diameter of $\Delta_{X}$ to be the maximum value of $d_{\Delta_{X}}(g, h)$, taken over all pairs of elements $g$ and $h$ of $X$, notably a finite set. We remark that $\Delta_{X}$ is connected if and only if its diameter is $<\infty$.

\subsection{History}

Commuting graphs were first studied by Y. Segev in the paper [11]. There the following concept was introduced, in advance of the main result 1.2.2.

Definition 1.2.1. Let $G$ be a nontrivial finite group. Then the commuting graph $\Delta_{G^{*}}$ is called balanced if there exist elements $g$ and $h$ in $G^{*}$ such that $d_{\Delta_{G^{*}}}(x, y) \geq 3$ for each pair $\{x, y\} \in\left\{\{g, h\},\{g, g h\},\{h, g h\},\left\{g, g^{-1} h\right\},\left\{h, g^{-1} h\right\}\right\}$.

Theorem 1.2.2 (Segev). Let $G$ be a nonabelian finite simple group, and $D$ a finite dimensional division algebra over an arbitrary field. If $\Delta_{G^{*}}$ is balanced or has diameter $>4$, then $G$ cannot be isomorphic to a quotient of the multiplicative group of $D$. 
In the subsequent paper [12], a complementary result was realized.

Theorem 1.2.3 (Segev, Seitz). If $G$ is a nonabelian finite simple group, then $\Delta_{G^{*}}$ is balanced or has diameter $>4$.

In combination, 1.2.2 and 1.2.3 resolved a conjecture of [7], having an unexpected relationship to commuting graphs and their diameters.

Conjecture 1.2.4 (Potapchik, Rapinchuk). Let D be a finite dimensional division algebra over an arbitrary field. Then no quotient of the multiplicative group of $D$ is a nonabelian finite simple group.

A further connection between division algebras and commuting graphs was exhibited in the following result of [8].

Theorem 1.2.5 (Rapinchuk, Segev). Let $D$ be a finite dimensional division algebra over a finitely generated field, and let $D^{\#}$ be the multiplicative group of $D$. Let $N$ be a normal subgroup of $D^{\#}$ of finite index. If $\Delta_{\left(D^{\#} / N\right)^{*}}$ has diameter $\geq 4$, then $N$ is open in $D$ with respect to a nontrivial (height one) valuation of $D$.

Commuting graphs were also key in realizing the culminating result in this line of research, found in [9].

Theorem 1.2.6 (Rapinchuk, Segev, Seitz). Let $D$ be a finite dimensional division algebra. Then any finite quotient of the multiplicative group of $D$ is solvable.

More recently, the study of commuting graphs has gained independent combinatorial interest. For example, the following result was proved in the paper [1]. 
Theorem 1.2.7 (Bates, Bundy, Perkins, Rowley). For positive integers $n \geq 2$ and $m \leq n / 2$, let $X_{m}$ be the set of all products of $m$ disjoint transpositions in the symmetric group $S_{n}$. Then $\Delta_{X_{m}}$ is disconnected if and only if $n=2 m+1$ or $n=4$ and $m=1$. Furthermore if $\Delta_{X_{m}}$ is connected, then the diameter of $\Delta_{X_{m}}$ is $\leq 3$, unless $2 m+2=n \in\{6,8,10\}$, in which case the diameter of $\Delta_{X_{m}}$ is 4.

In the follow-up paper [2], Bundy examines the commuting graph $\Delta_{X}$ for an arbitrary conjugacy class $X \subseteq S_{n}$. In particular, a necessary and sufficient condition for connectivity of $\Delta_{X}$ is given, based on the cycle structure of $X$.

\subsection{Overview}

The main contribution of this dissertation, in Chapter 2, is a complete classification of the structure of $\Delta_{S_{n}^{*}}$, based on the nature of $n$. In particular each connected component is identified, along with its diameter. Especially noteworthy is that regardless of the value of $n$, no component has diameter $>5$. In fact the typical case is that $\Delta_{S_{n}^{*}}$ is connected of diameter 5. Among our arguments, we develop a construction that yields a new proof of a result first obtained by Bates et al., in their paper [1]. We remark that the alternating groups $A_{n}$ were explored by Segev and Seitz in [12]. But only a lower bound on the diameter of $\Delta_{A_{n}^{*}}$ was given, and moreover our approach to understanding $\Delta_{S_{n}^{*}}$ is essentially different, and more elementary.

In Chapter 3, we show that if $G$ is a finite group with a nontrivial abelian normal 
subgroup, and if for all primes $p$ dividing the order of $G$, each Sylow $p$-subgroup of $G$ has multiple subgroups of order $p$, then $\Delta_{G^{*}}$ is connected of diameter $\leq 7$. We remark that the structure possibilities for a finite $p$-group with only a single subgroup of order $p$ are completely understood. In particular, such a group is either cyclic, or a generalized quaternion group. A more detailed discussion of this classification is given in Chapter 3, or one may consult [10, pp.141-143]. We note that if $G$ is a nontrivial finite solvable group with derived series $G=G_{0} \supsetneq G_{1} \supsetneq \cdots \supsetneq G_{i-1} \supsetneq G_{i}=\left\{1_{G}\right\}$, then $G_{i-1}$ is a nontrivial abelian normal subgroup of $G$. (Refer to the proof of 3.1.3.) Thus as a corollary to the chapter's main result, we realize an absolute bound on the diameter of $\Delta_{G^{*}}$, for a solvable group with no cyclic or generalized quaternion Sylow subgroups.

Chapter 4 is an investigation of groups whose order is divisible by exactly two distinct primes. If $G$ is a group of order $p^{a} q^{b}$, where $p$ and $q$ are prime and $p \neq q$, then $G$ is solvable by Burnside's Theorem. Thus the bound of Chapter 3 applies to the diameter of $\Delta_{G^{*}}$, provided that the Sylow $p$ - and $q$-subgroups are suitable. However, we give a precise condition for connectivity. In particular, the bound of 7 on the diameter of $\Delta_{G^{*}}$ applies as long as $G$ possesses an element of mixed order, that is, of order divisible by both $p$ and $q$. But if $G$ is comprised of $p$ - and $q$-elements only, then $\Delta_{G^{*}}$ is disconnected. For disconnected cases, we give a complete description of the structure of the group $G$. 


\section{Chapter 2}

\section{Symmetric Groups}

\subsection{Main result}

Definition 2.1.1. For a positive integer $n$, we define the symmetric group on $\mathbf{n}$ elements, denoted $\mathbf{S}_{\mathbf{n}}$, to be the set of all bijective functions on the set $\{1,2, \ldots, n\}$. For $n \geq 2$ and $m \in\{2,3, \ldots, n\}$, we let $\mathbf{C}_{\mathbf{n}}^{\mathbf{m}}$ denote the set of all cycles of length $m$ in $S_{n}$. Also, we define $\mathbf{R}_{\mathbf{n}}=S_{n}^{*} \backslash\left(C_{n}^{n} \cup C_{n}^{n-1}\right)$.

Theorem 2.1.2. Suppose that $n \geq 3$, so that $\Delta_{S_{n}^{*}}$ is nontrivial.

a. If $n-1$ and $n$ are composite numbers, then $\Delta_{S_{n}^{*}}$ is connected of diameter 5 .

b. If $n-1$ is a prime, then $\Delta_{S_{n}^{*}}$ has connected components $\Delta_{R_{n} \cup C_{n}^{n}}$, and $\Delta_{\langle\gamma\rangle^{*}}$, $\gamma \in C_{n}^{n-1}$. The diameter of $\Delta_{R_{n} \cup C_{n}^{n}}$ is 1, 3, or 4 according as $n$ is 3, 4, or $>4$. The diameter of each $\Delta_{\langle\gamma\rangle^{*}}$ is 0 or 1 , according as $n$ is 3 or $>3$.

c. If $n$ is a prime, $n>3$, then $\Delta_{S_{n}^{*}}$ has connected components $\Delta_{R_{n} \cup C_{n}^{n-1}}$, and $\Delta_{\langle\gamma\rangle^{*}}, \gamma \in C_{n}^{n}$. The diameter of $\Delta_{R_{n} \cup C_{n}^{n-1}}$ is 5 ; the diameter of each $\Delta_{\langle\gamma\rangle^{*}}$ is 1 . 
We shall realize the theorem through a number of sections. For more on $S_{n}$, refer to $[4$, p.46], for instance. Throughout the chapter, $n$ denotes a positive integer.

\subsection{Commuting elements of $S_{n}$}

The results developed here are well known, but included for completeness.

Definition 2.2.1. Suppose that $\pi$ and $\rho$ are elements of $S_{n}$. We say that $\pi$ induces a cycle map on $\rho$ if, for each cycle $\left(\begin{array}{lllll}a_{0} & a_{1} & \cdots & a_{i-1}\end{array}\right)$ in the decomposition of $\rho$, $\left(\pi\left(a_{0}\right) \pi\left(a_{1}\right) \cdots \pi\left(a_{i-1}\right)\right)$ is a cycle of $\rho$ as well.

Proposition 2.2.2. Let $\pi$ and $\rho$ be elements of $S_{n}$. Then $\pi$ and $\rho$ commute if and only if $\pi$ induces a cycle map on $\rho$.

Proof. Suppose that $\pi$ and $\rho$ are commuting elements, and let $\left(a_{0} a_{1} \cdots a_{i-1}\right)$ be a cycle of $\rho$, possibly with $i=1$. Then $\rho\left(a_{j}\right)=a_{(j+1) \bmod i}$ for $0 \leq j<i$. Therefore,

$$
\rho\left(\pi\left(a_{j}\right)\right)=\pi\left(\rho\left(a_{j}\right)\right)=\pi\left(a_{(j+1) \bmod i}\right)
$$

Hence $\left(\pi\left(a_{0}\right) \pi\left(a_{1}\right) \cdots \pi\left(a_{i-1}\right)\right)$ is a cycle of $\rho$.

Now assume that $\pi$ induces a cycle map on $\rho$, and let $a \in\{1,2, \ldots, n\}$. Suppose that $\left(a_{0} a_{1} \cdots a_{i-1}\right)$ is the cycle of $\rho$ containing $a$; assume that $a_{j}=a$. We observe that $\rho\left(a_{j}\right)=a_{(j+1) \bmod i}$, thus $(\pi \rho)\left(a_{j}\right)=\pi\left(a_{(j+1) \bmod i}\right)$. Since $\pi$ induces a cycle map on $\rho,\left(\pi\left(a_{0}\right) \pi\left(a_{1}\right) \cdots \pi\left(a_{i-1}\right)\right)$ is a cycle of $\rho$. Thus $\rho\left(\pi\left(a_{j}\right)\right)=\pi\left(a_{(j+1) \bmod i}\right)$, in particular. Therefore $(\pi \rho)\left(a_{j}\right)=(\rho \pi)\left(a_{j}\right)$. We conclude that $\pi$ and $\rho$ are commuting elements, because $a$ was chosen arbitrarily from $\{1,2, \ldots, n\}$, and $a_{j}=a$. 
We remark that the statement of 2.2.2 is symmetric with respect to $\pi$ and $\rho$. Therefore, we realize that $\pi$ induces a cycle map on $\rho$ if and only if $\rho$ induces a cycle map on $\pi$.

Proposition 2.2.3. Let $\pi \in S_{n}$, and let $\gamma$ be a cycle in the decomposition of $\pi$. Then $\pi$ commutes with $\gamma$.

Proof. Suppose that $\gamma=\left(a_{0} a_{1} \cdots a_{i-1}\right)$. Then for $0 \leq j<i$, we have $\pi\left(a_{j}\right)=\gamma\left(a_{j}\right)$, because $\gamma$ is a cycle of $\pi$. Therefore,

$$
\left(\pi\left(a_{0}\right) \pi\left(a_{1}\right) \cdots \pi\left(a_{i-1}\right)\right)=\left(a_{1} a_{2} \cdots a_{i-1} a_{0}\right)=\gamma
$$

Hence $\pi$ induces a cycle map on $\gamma$, and so $\pi$ and $\gamma$ commute by 2.2 .2 .

Proposition 2.2.4. Suppose that $\pi$ and $\rho$ are commuting elements of $S_{n}$, and let $\gamma=\left(a_{0} a_{1} \cdots a_{i-1}\right)$ be a cycle in the decomposition of $\pi$. Assume that $\gamma$ has unique length among all of cycles of $\pi$. Then $\rho$ acts on $\left\{a_{0}, a_{1}, \ldots, a_{i-1}\right\}$ as a power of $\gamma$.

Proof. Since $\pi$ and $\rho$ commute, $\left(\rho\left(a_{0}\right) \rho\left(a_{1}\right) \cdots \rho\left(a_{i-1}\right)\right)$ is a cycle of $\pi$ by 2.2.2. Therefore $\left(\rho\left(a_{0}\right) \rho\left(a_{1}\right) \cdots \rho\left(a_{i-1}\right)\right)=\gamma$, because of the unique length of $\gamma$. Assuming that $\rho\left(a_{0}\right)=a_{k}$, we observe that $\gamma$ may also be expressed as

$$
\left(a_{k} a_{(k+1) \bmod i} \cdots a_{(k+i-1) \bmod i}\right) .
$$

Hence we conclude that $\rho\left(a_{j}\right)=a_{(k+j) \bmod i}$ for $0 \leq j<i$. In other words, $\rho$ acts on $\left\{a_{0}, a_{1}, \ldots, a_{i-1}\right\}$ in precisely the same manner as $\gamma^{k}$ 
Definition 2.2.5. Let $\pi \in S_{n}$. We define the fixed set of $\pi$ to be the set of all elements $a \in\{1,2, \ldots, n\}$ such that $\pi(a)=a$. We denote this set by $F(\pi)$.

Proposition 2.2.6. Suppose that $\pi, \rho \in S_{n}$ are commuting elements. Also assume that $F(\pi)$ contains exactly one element, say a. Then $a \in F(\rho)$ as well.

Proof. We observe that the element $a$ constitutes a trivial cycle of $\pi$, since $a \in F(\pi)$. Furthermore this is true of the element $\rho(a)$, by 2.2.2. But $\pi$ has only one trivial cycle, because $F(\pi)$ contains just a single element. Hence we conclude that $\rho(a)=a$.

Definition 2.2.7. Let $\pi \in S_{n}$. We define the moved set of $\pi$ to be complement of the fixed set of $\pi$, with respect to $\{1,2, \ldots, n\}$. We denote the moved set by $M(\pi)$.

Definition 2.2.8. Let $\pi, \rho \in S_{n}$. We say that $\pi$ and $\rho$ are disjoint elements if $M(\pi) \cap M(\rho)$ is empty.

Proposition 2.2.9. Assume that $\pi$ and $\rho$ are disjoint elements of $S_{n}$. Then $\pi$ and $\rho$ commute.

Proof. Since $\pi$ and $\rho$ are disjoint, $\pi$ acts as the identity on $M(\rho)$. Thus $\pi$ induces a function on $F(\rho)$. Likewise, $\rho$ acts on $F(\pi)$. Now let $a \in\{1,2, \ldots, n\}$. We observe that $a$ is fixed by $\pi$ or $\rho$, because $\pi$ and $\rho$ are disjoint elements. If $a \in F(\pi)$, then $(\rho \pi)(a)=\rho(a)$. Moreover we have $(\pi \rho)(a)=\rho(a)$, since $\rho(a) \in F(\pi)$ as well. Hence $(\rho \pi)(a)=(\pi \rho)(a)$. This conclusion is drawn similarly if $a \in F(\rho)$; thus $(\rho \pi)(a)=(\pi \rho)(a)$ holds in general. Therefore $\pi$ and $\rho$ are commuting elements. 
Definition 2.2.10. Let $H$ be a subgroup of $S_{n}$. For an element $a \in\{1,2, \ldots, n\}$, we define its orbit under the natural action of $\mathbf{H}$ to be the set $\{\pi(a) \mid \pi \in H\}$. We denote this set by $[a]_{H}$.

Proposition 2.2.11. Let $H$ be a subgroup of $S_{n}$. Then the family of all orbits under the natural action of $H,\left\{[a]_{H} \mid a \in\{1,2, \ldots, n\}\right\}$, forms a partition of $\{1,2, \ldots, n\}$ such that $a \in[a]_{H}$ for all $a \in\{1,2, \ldots, n\}$.

Proof. Since $H$ is a subgroup of $S_{n}, H$ contains the identity function. Hence, for $a \in\{1,2, \ldots, n\}$, we have $a \in[a]_{H}$. Suppose $b \in\{1,2, \ldots, n\}$ as well, and assume that $[a]_{H} \cap[b]_{H}$ is nonempty. Select $x \in[a]_{H} \cap[b]_{H}$, and suppose that $\pi(a)=\rho(b)=x$, where $\pi, \rho \in H$. Furthermore let $y \in[a]_{H}$, and assume that $y=\varphi(a)$, where $\varphi \in H$. Then we have

$$
\left(\varphi \pi^{-1} \rho\right)(b)=\left(\varphi \pi^{-1}\right)(x)=\varphi(a)=y
$$

Also, $\varphi \pi^{-1} \rho \in H$, because $H$ is a group. Therefore $y \in[b]_{H}$, hence $[a]_{H} \subseteq[b]_{H}$. And the reverse containment is established by analogy. Thus $[a]_{H}=[b]_{H}$.

Proposition 2.2.12. Let $H$ be a subgroup of $S_{n}$. Assume that $\pi$ is a member of the center of $H$, and suppose that $a \in F(\pi)$. Then $[a]_{H} \subseteq F(\pi)$.

Proof. Let $b \in[a]_{H}$, and suppose that $\rho(a)=b$, where $\rho \in H$. Since $\pi$ is an element of the center of $H, \pi$ and $\rho$ are commuting elements. Therefore,

$$
\pi(b)=\pi(\rho(a))=\rho(\pi(a))=\rho(a)=b .
$$

The lemma follows. 


\subsection{The commuting graph $\Delta_{R_{n}}$}

Theorem 2.3.1. Suppose that $n \geq 4$. Then $\Delta_{R_{n}}$ is connected. Furthermore, the diameter of $\Delta_{R_{n}}$ is 3 or 4 , according as $n=4$ or $n>4$.

The proof of the theorem is realized via a sequence of results. We remark that $R_{n}$ is empty for $n \in\{1,2,3\}$.

Proposition 2.3.2. The diameter of $\Delta_{R_{4}}$ is 3 .

Proof. Let us define

$$
\begin{aligned}
T & =\{(12),(13),(14),(23),(24),(34)\} \\
D & =\{(12)(34),(13)(24),(14)(23)\}
\end{aligned}
$$

We observe that $T$ is the set of all transpositions in $S_{4}$, and $D$ is the set of all double transpositions. Also, any element of $S_{4}^{*}$ that is neither a cycle of length 3 nor a cycle of length 4 is a member of $T \cup D$. Therefore $R_{4}=T \cup D$.

We claim that any $\pi \in R_{4}$ commutes with an element $\varphi \in D$. If $\pi \in D$, we may take $\varphi=\pi$, because a group element commutes with itself. Given that $\pi \in T$, let us assume that $\pi=(a b)$. Then by $2.2 .3, \pi$ commutes with $\varphi=(a b)(c d) \in D$, where $c$ and $d$ are the elements of $\{1,2,3,4\} \backslash\{a, b\}$ in arbitrary order. Thus we have our claim. Moreover we realize that the elements of $D$ mutually commute. For it is easily seen that each member of $D$ induces a cycle map on each of the other members; hence we appeal to 2.2 .2 . 
Let $\pi$ and $\varphi$ be as above, and let $\rho \in R_{4}$ as well. Also, assume that $\psi \in D$ commutes with $\rho$. Then $(\pi, \varphi, \psi, \rho)$ is a commuting path in $\Delta_{R_{4}}$, because $\varphi$ and $\psi$ commute. Therefore $d_{\Delta_{R_{4}}}(\pi, \rho) \leq 3$, and so the diameter of $\Delta_{R_{4}}$ is at most 3 .

Now consider the particular pair of elements $\sigma=\left(\begin{array}{ll}1 & 2\end{array}\right)$ and $\tau=\left(\begin{array}{ll}1 & 3\end{array}\right)$ of $R_{4}$. Suppose that $\chi \in S_{4}$ commutes $\sigma$ and $\tau$. Then by 2.2.2, $\chi$ induces a cycle map on $\sigma$, and likewise on $\tau$. Thus $\chi$ maps the set $\{1,2\}$ to itself, and the same goes for $\{1,3\}$. It follows that $\{1,2,3\} \subseteq F(\chi)$. Hence $\chi$ induces a function on $\{1,2,3,4\} \backslash\{1,2,3\}$; that is, $4 \in F(\chi)$. We conclude that $\chi$ is the identity element of $S_{4}$. Hence there is no commuting path of the form $(\sigma, \chi, \tau)$ in $\Delta_{S_{4}^{*}}$. Therefore $d_{\Delta_{S_{4}^{*}}}(\sigma, \tau) \geq 3$, which implies that $d_{\Delta_{R_{4}}}(\sigma, \tau) \geq 3$. Thus, the diameter of $\Delta_{R_{4}}$ is at least 3 .

Lemma 2.3.3. Suppose that $n>4$, and let $\pi \in R_{n}$. Then $\pi$ is a product of two disjoint cycles, each of length $n / 2$, or $\pi$ commutes with a nontrivial cycle of length $<n / 2$. In any case, $\pi$ commutes with a nontrivial cycle of length $\leq n / 2$.

Proof. First assume that $\pi$ is itself a cycle. Then by the definition of $R_{n}, \pi$ fixes at least two elements, say $a, b \in\{1,2, \ldots, n\}$. We observe that $\pi$ commutes with $(a b)$, by 2.2.9. Also the length of $(a b)$, namely 2 , is $<n / 2$ because $n>4$.

Now assume that $\pi$ is neither a cycle, nor a product of two disjoint cycles of length $n / 2$ each. Let $\gamma$ be a nontrivial cycle of minimum length over the decomposition of $\pi$. Then the length of $\gamma$ is obviously $<n / 2$. And $\gamma$ commutes with $\pi$, by 2.2.3.

Finally, if the decomposition of $\pi$ consists of two disjoint cycles, each of length $n / 2$, then by $2.2 .3, \pi$ commutes with at least two nontrivial cycles of length $\leq n / 2$. 
Proposition 2.3.4. For $n>4$, the diameter of $\Delta_{R_{n}}$ is $\leq 4$.

Proof. Let $\pi, \rho \in R_{n}$. First suppose that at least one of $\pi$ and $\rho$, say $\pi$, commutes with a cycle $\gamma \in R_{n}$ of length $<n / 2$. We observe that $\rho$ commutes with a cycle $\delta \in R_{n}$ of length $\leq n / 2$, by 2.3.3. If $\gamma$ and $\delta$ are disjoint, on the one hand, then these cycles commute by 2.2.9. Thus $(\pi, \gamma, \delta, \rho)$ is a commuting path in $\Delta_{R_{n}}$, and so $d_{\Delta_{R_{n}}}(\pi, \rho) \leq 3$. On the other hand if $M(\gamma) \cap M(\delta)$ is nonempty, then

$$
|M(\gamma) \cup M(\delta)| \leq|M(\gamma)|+|M(\delta)|-1<2(n / 2)-1=n-1
$$

Therefore $F(\gamma) \cap F(\delta)$ contains at least two elements, say $a$ and $b$. We define $\sigma=(a b) \in R_{n}$, and observe that $\sigma$ commutes with $\gamma$ and $\delta$ by 2.2.9. Moreover, $(\pi, \gamma, \sigma, \delta, \rho)$ is a commuting path in $\Delta_{R_{n}}$. Thus $d_{\Delta_{R_{n}}}(\pi, \rho) \leq 4$.

Now assume that neither $\pi$ nor $\rho$ commutes with a cycle of length $<n / 2$. Then $\pi$ is a product of two disjoint cycles, each of length $n / 2$, by 2.3.3. Likewise, this is true of $\rho$. Assume that

$$
\begin{aligned}
& \pi=\left(\begin{array}{llll}
a_{1} & a_{2} & \cdots & a_{n / 2}
\end{array}\right)\left(\begin{array}{llll}
b_{1} & b_{2} & \cdots & b_{n / 2}
\end{array}\right), \\
& \rho=\left(\begin{array}{llll}
c_{1} & c_{2} & \cdots & c_{n / 2}
\end{array}\right)\left(\begin{array}{llll}
d_{1} & d_{2} & \cdots & d_{n / 2}
\end{array}\right) .
\end{aligned}
$$

Since $M(\pi)=M(\rho)=\{1,2, \ldots, n\}$, we may take $a_{1}=c_{1}$. Therefore there exists $i \in\{1,2, \ldots, n / 2\}$ such that $b_{i} \notin\left\{c_{j} \mid 1 \leq j \leq n / 2\right\}$. Hence $b_{i} \in\left\{d_{j} \mid 1 \leq j \leq n / 2\right\}$, 
and thus we may suppose that $b_{1}=d_{1}$. Let us define

$$
\begin{aligned}
\varphi & =\left(a_{1} b_{1}\right)\left(a_{2} b_{2}\right) \cdots\left(a_{n / 2} b_{n / 2}\right), \\
\tau & =\left(a_{1} b_{1}\right)=\left(c_{1} d_{1}\right), \\
\psi & =\left(c_{1} d_{1}\right)\left(c_{2} d_{2}\right) \cdots\left(c_{n / 2} d_{n / 2}\right) .
\end{aligned}
$$

Then obviously $\varphi, \tau$, and $\psi$ are elements of $R_{n}$. Moreover we claim that $(\pi, \varphi, \tau, \psi, \rho)$ is a commuting path in $\Delta_{R_{n}}$. We have

$$
\begin{aligned}
\left(\varphi\left(a_{1}\right) \varphi\left(a_{2}\right) \cdots \varphi\left(a_{n / 2}\right)\right) & =\left(b_{1} b_{2} \cdots b_{n / 2}\right) \\
\left(\varphi\left(b_{1}\right) \varphi\left(b_{2}\right) \cdots \varphi\left(b_{n / 2}\right)\right) & =\left(a_{1} a_{2} \cdots a_{n / 2}\right) .
\end{aligned}
$$

Thus $\varphi$ induces a cycle map on $\pi$; hence $\varphi$ and $\pi$ are commuting elements by 2.2.2. This similarly holds for $\psi$ and $\rho$. And $\tau$, being a cycle in the decomposition of both $\varphi$ and $\psi$, commutes with each of these elements by 2.2.3. Therefore we have our claim; hence $d_{\Delta_{R_{n}}}(\pi, \rho) \leq 4$. And via our cases, we have now shown that this bound holds for a general pair $\pi, \rho \in R_{n}$. Thus we have the proposition.

Proposition 2.3.5. Suppose $n>4$. Let $m$ be the odd element of the set $\{n-1, n\}$, and define

$$
\pi=(12 \cdots m-2)(m-1 m) \in R_{n}, \quad \rho=(23 \cdots m-1)(1 m) \in R_{n} .
$$

Then $d_{\Delta_{S_{n}^{*}}}(\pi, \rho) \geq 4$.

Proof. Suppose that $d_{\Delta_{S_{n}^{*}}}(\pi, \rho) \leq 3$. Let $(\pi, \varphi, \psi, \rho)$ be a commuting path in $\Delta_{S_{n}^{*}}$. We observe that $m \geq 5$; hence the cycles of $\pi$, including a trivial one if $n$ is even, 
have distinct lengths. Therefore by 2.2.4, there exist positive integers $r$ and $s$ such that

$$
\varphi=(12 \cdots m-2)^{r}(m-1 m)^{s} .
$$

Similarly, there exist $t, u \in \mathbb{N}$ such that

$$
\psi=(23 \cdots m-1)^{t}(1 m)^{u} .
$$

We also note that $\varphi$ and $\psi$ are nontrivial, being elements of $S_{n}^{*}$. Moreover they are commuting elements, by our assumption that they appear consecutively in a commuting path.

Since $m-2$ is odd, the cycle decomposition of $(12 \cdots m-2)^{r}$ does not contain a transposition. Therefore $(m-1 m)$ is the only potential transposition among the cycles of $\varphi$. So, if $(m-1 m)$ is a cycle of $\varphi$, then $(\psi(m-1) \psi(m))=(m-1 m)$ by 2.2.2. But clearly $\psi(m-1) \neq m$. Thus $m-1$ and $m$ are fixed by $\psi$. However, this implies that $\psi$ is the identity on all of $\{1,2, \ldots, n\}$, contradicting that $\psi$ is nontrivial. Hence $(m-1 m)$ must not be a cycle of $\varphi$, and we therefore conclude that $m-1$ and $m$ are fixed by $\varphi$. By a parallel argument, $\psi$ fixes the elements 1 and $m$. Thus

$$
\varphi=(12 \cdots m-2)^{r}, \quad \psi=(23 \cdots m-1)^{t} .
$$

Now on the one hand, we observe that $\varphi(1) \in\{2,3, \ldots, m-2\}$, because $\varphi$ is nontrivial. On the other hand, since $\varphi$ and $\psi$ commute, and $\psi^{-1}$ fixes 1 , we have $\varphi(1)=\left(\psi \varphi \psi^{-1}\right)(1)=\psi(\varphi(1))$. Therefore $\psi$ fixes an element of $\{2,3, \ldots, m-2\}$. But then $\psi=\mathrm{id}$, a contradiction. 
We may now give an argument for Theorem 2.3.1.

Proof. We obtain the result by combining 2.3.2, 2.3.4, and 2.3.5. We note that 2.3.5 implies that $\pi$ and $\rho$ are at distance $\geq 4$ in $\Delta_{R_{n}}$, a subgraph of $\Delta_{S_{n}^{*}}$.

\subsection{The commuting graph $\Delta_{R_{n} \cup C_{n}^{n}}$ for even $n$}

We shall prove that if $n$ is even, the result of Theorem 2.3.1 applies to the larger graph $\Delta_{R_{n} \cup C_{n}^{n}}$

Theorem 2.4.1. Suppose that $n$ is even, $n \geq 4$. Then $\Delta_{R_{n} \cup C_{n}^{n}}$ is connected. Moreover the diameter of the commuting graph is 3 or 4 , according as $n=4$ or $n>4$.

Once again, the theorem is realized through several propositions.

Proposition 2.4.2. The diameter of $\Delta_{R_{4} \cup C_{4}^{4}}$ is 3 .

Proof. From the proof of 2.3.2, we recall that $D$ denotes the set of all double transpositions in $S_{4}$. In the proof we argued that $\Delta_{R_{4}}$ has diameter $\leq 3$ through two observations. In particular, the elements of $D$ mutually commute, and each element of $R_{4}$ commutes with an element of $D$. The second of these observations extends to include $C_{4}^{4}$. Indeed, given $\gamma=\left(\begin{array}{llll}a & b & c & d\end{array}\right) \in C_{4}^{4}$, we observe that $\gamma$ commutes with $\gamma^{2}=(a c)(b d) \in D$. Thus, as in the proof of 2.3.2, we conclude that $\Delta_{R_{4} \cup C_{4}^{4}}$ has diameter $\leq 3$. 
Now, in demonstrating that the inequality of 2.3.2 is sharp, we defined $\sigma=\left(\begin{array}{ll}1 & 2\end{array}\right)$ and $\tau=(23)$, elements of $R_{4}$, and argued that $d_{\Delta_{S_{4}^{*}}}(\sigma, \tau) \geq 3$. Thus the distance between $\sigma$ and $\tau$ is $\geq 3$ in $\Delta_{R_{4} \cup C_{4}^{4}}$. We conclude that $\Delta_{R_{4} \cup C_{4}^{4}}$ has diameter $\geq 3$.

Lemma 2.4.3. Suppose that $n \geq 4$. Let $\pi \in R_{n}$ be a product of $i \geq 2$ disjoint nontrivial cycles of a common length. Let $\gamma \in R_{n}$ be a nontrivial cycle of length $j$, where $j \leq i$. Then there exists an element $\rho \in R_{n}$ that commutes with $\pi$ and $\gamma$.

Proof. We consider two possibilities. First assume that there exists a nontrivial cycle $\delta$ in the decomposition of $\pi$ that is disjoint from $\gamma$. Then $\pi$ and $\delta$ commute, by 2.2.3, and $\gamma$ and $\delta$ commute as well, by 2.2.9. We observe that $\delta$ is an element of $R_{n}$, because its length is at most $n / i \leq n / 2 \leq n-2$. Thus $\delta$ may serve as $\rho$.

Now let us assume that for each nontrivial cycle $\delta$ in the decomposition of $\pi$, $M(\gamma) \cap M(\delta)$ is nonempty. Then the number of nontrivial cycles of $\pi$ does not exceed the length of $\gamma$. In other words $i \leq j$, and hence $i=j$, because the reverse inequality is being assumed. Let $m$ denote the common length of the cycles of $\pi$, and suppose that in decomposed form we have

$$
\pi=\prod_{k=0}^{i-1}\left(a_{k, 0} a_{k, 1} a_{k, 2} \cdots a_{k, m-1}\right)
$$

Also suppose that $\gamma=\left(b_{0} b_{1} b_{2} \cdots b_{i-1}\right)$, and with no loss in generality, take $a_{k, 0}=b_{k}$ for $0 \leq k<i$. Define

$$
\rho=\prod_{l=0}^{m-1}\left(a_{0, l} a_{1, l} a_{2, l} \cdots a_{i-1, l}\right) .
$$


We observe that $\rho$ is nontrivial, because $i \geq 2$, and not itself a cycle, because $m \geq 2$. Hence $\rho \in R_{n}$. Also, $\rho$ commutes with $\left(\begin{array}{lllll}a_{0,0} & a_{1,0} & a_{2,0} & \cdots & a_{i-1,0}\end{array}\right)$, one of its cycles, by 2.2.3. Thus $\rho$ commutes with $\gamma$. Furthermore, for each $k \in\{0,1, \ldots, i-1\}$, and each $l \in\{0,1, \ldots, m-1\}$,

$$
\begin{aligned}
& (\rho \pi)\left(a_{k, l}\right)=\rho\left(a_{k,(l+1) \bmod m}\right)=a_{(k+1) \bmod i,(l+1) \bmod m} \\
& (\pi \rho)\left(a_{k, l}\right)=\pi\left(a_{(k+1) \bmod i, l}\right)=a_{(k+1) \bmod i,(l+1) \bmod m} .
\end{aligned}
$$

Hence $\rho$ commutes with $\pi$ as well, because both of $\pi$ and $\rho$ fix each element of the set

$$
\{1,2, \ldots, n\} \backslash\left\{a_{k, l} \mid 0 \leq k<i, 0 \leq l<m\right\}
$$

Proposition 2.4.4. Suppose that $n$ is even, $n>4$. Let $\pi \in R_{n}$, and $\gamma \in C_{n}^{n}$. Then the distance between $\pi$ and $\gamma$ in $\Delta_{R_{n} \cup C_{n}^{n}}$ is $\leq 4$.

Proof. Assume that $\gamma=\left(\begin{array}{llll}a_{1} & a_{2} & \cdots & a_{n}\end{array}\right)$. Since $n$ is even, we have

$$
\gamma^{n / 2}=\left(a_{1} a_{n / 2+1}\right)\left(a_{2} a_{n / 2+2}\right) \cdots\left(a_{n / 2} a_{n}\right) \in R_{n}
$$

We observe that $\gamma$ commutes with $\gamma^{n / 2}$, because a group element commutes with each of its powers. By 2.3.3, there exists a nontrivial cycle $\delta \in R_{n}$ of length $\leq n / 2$ that commutes with $\pi$, because $\pi \in R_{n}$. Furthermore by 2.4.3, there exists $\rho \in R_{n}$ commuting with $\gamma^{n / 2}$ and $\delta$. Hence $\left(\pi, \delta, \rho, \gamma^{n / 2}, \gamma\right)$ is a commuting path in $\Delta_{R_{n}} \cup C_{n}^{n}$. Therefore we have the proposition. 
Lemma 2.4.5. Suppose that $n$ is even, and let $\pi \in S_{n}$ be a product of $n / 2$ disjoint transpositions. Then the order of the centralizer of $\pi$ in $S_{n}$ is given by

$$
(n / 2) ! \cdot 2^{n / 2}=(2)(4)(6)(8) \cdots(n)
$$

Proof. In view of 2.2.2, we must show that the number of elements of $S_{n}$ that induce a cycle map on $\pi$ is $(n / 2) ! \cdot 2^{n / 2}$. Suppose that we have the decomposition

$$
\pi=\left(a_{1} b_{1}\right)\left(a_{2} b_{2}\right) \cdots\left(a_{n / 2} b_{n / 2}\right)
$$

Consider a general element of $S_{n}$, written in table form as follows:

$$
\rho=\left(\begin{array}{ccccccc}
a_{1} & b_{1} & a_{2} & b_{2} & \cdots & a_{n / 2} & b_{n / 2} \\
x_{1} & y_{1} & x_{2} & y_{2} & \cdots & x_{n / 2} & y_{n / 2}
\end{array}\right)
$$

We observe that $\rho$ induces a cycle map on $\pi$ if and only if

$$
\left\{\left\{x_{1}, y_{1}\right\},\left\{x_{2}, y_{2}\right\}, \ldots\left\{x_{n / 2}, y_{n / 2}\right\}\right\}=\left\{\left\{a_{1}, b_{1}\right\},\left\{a_{2}, b_{2}\right\}, \ldots\left\{a_{n / 2}, b_{n / 2}\right\}\right\}
$$

To produce a table $\rho$ that satisfies this condition, we may first arrange the sets $\left\{a_{i}, b_{i}\right\}$, $1 \leq i \leq n / 2$, into a sequence, then arbitrarily order the pair of elements within each set. The number of ways to complete this process is

$$
(n / 2) ! \cdot 2^{n / 2}=(1 \cdot 2)(2 \cdot 2)(3 \cdot 2) \cdots((n / 2) \cdot 2)=(2)(4)(6) \cdots(n) .
$$

Proposition 2.4.6. Suppose that $n$ is even, $n>4$. For $\gamma, \delta \in C_{n}^{n}$, the distance between $\gamma$ and $\delta$ in $\Delta_{R_{n} \cup C_{n}^{n}}$ is $\leq 4$. 
Proof. Let $\pi=\gamma^{n / 2}$, and $\rho=\delta^{n / 2}$. We observe that $\gamma$ commutes with $\pi$, because a group element commutes with each of its powers. We also note that $\pi$ is a product of $n / 2$ disjoint transpositions, and in particular, $\pi \in R_{n}$. Likewise, $\delta$ commutes with $\rho \in R_{n}$, a product of $n / 2$ disjoint transpositions. Let $H$ and $K$ be the centralizers of $\pi$ and $\rho$ in $S_{n}$, respectively. By a well-known result of finite group theory, we have

$$
|H K|=\frac{|H| \cdot|K|}{|H \cap K|} .
$$

(Refer to [4, p.39].) It is also well known that $\left|S_{n}\right|=n !$. (See $[5$, p.32].) Therefore $|H K| \leq n !$, because $H K \subseteq S_{n}$. But by 2.4.5,

$$
|H| \cdot|K|=[(2)(4)(6) \cdots(n)]^{2}>n !
$$

Hence $|H \cap K|>1$. Let $\varphi \in(H \cap K) \backslash\{\mathrm{id}\}$, and note that $\varphi$ commutes with $\pi$ and $\rho$. Suppose that $\varphi \in C_{n}^{n-1}$. Then $F(\varphi)$ contains precisely one element, say $a$. Therefore by 2.2.6, $a \in F(\pi) \cap F(\rho)$. However $F(\pi)=F(\rho)=\emptyset$, obviously. Thus $\varphi \notin C_{n}^{n-1}$, and so $\varphi \in R_{n} \cup C_{n}^{n}$. We now realize that $(\gamma, \pi, \varphi, \rho, \delta)$ is a commuting path in $\Delta_{R_{n} \cup C_{n}^{n}}$. The proposition follows.

We may now provide an argument for Theorem 2.4.1.

Proof. The assertion for $n=4$ is handled in Proposition 2.4.2. For $n>4$ and $n$ even, we combine the results of 2.3.4, 2.3.5, 2.4.4, and 2.4.6.

We conclude the section by developing a second argument for Proposition 2.4.6, one which is more enlightening but also more technical. We shall illustrate the construction of a particular element $\varphi$, based on $\pi=\gamma^{n / 2}$ and $\rho=\delta^{n / 2}$. Especially 
noteworthy is that the element $\varphi$, like $\pi$ and $\rho$, will be a product of $n / 2$ disjoint transpositions.

Lemma 2.4.7. Suppose that $n$ is even, $n \geq 4$. Let $\pi, \rho \in S_{n}$. Furthermore assume that each of $\pi$ and $\rho$ is a product of $n / 2$ disjoint transpositions. Then for all nonnegative integers $j$,

$$
F\left[\pi(\rho \pi)^{j}\right]=F\left[\rho(\pi \rho)^{j}\right]=\emptyset
$$

Proof. We proceed by induction on $j$. Clearly we have $F(\pi)=F(\rho)=\emptyset$, thus (2.4.2) holds for $j=0$. Let $j$ be a nonnegative integer, and inductively assume that (2.4.2) holds for this particular $j$. However, suppose that $F\left[\pi(\rho \pi)^{j+1}\right] \neq \emptyset$. Then there exists an element $a \in\{1,2, \ldots, n\}$ such that $\left[\pi(\rho \pi)^{j+1}\right](a)=a$. We note that $\pi^{-1}=\pi$, because $\pi$ is a product of disjoint transpositions. Therefore $\left[\rho(\pi \rho)^{j}\right](\pi(a))=\pi(a)$, and hence $F\left[\rho(\pi \rho)^{j}\right]$ is nonempty. This is a contradiction. Thus $F\left[\pi(\rho \pi)^{j+1}\right]=\emptyset$. And by a parallel argument, $F\left[\rho(\pi \rho)^{j+1}\right]=\emptyset$. Hence we have the lemma, by induction.

Lemma 2.4.8. Let $n, \pi$, and $\rho$ be as in Lemma 2.4.7. Let $H$ be the subgroup of $S_{n}$ generated by $\pi$ and $\rho$, and let $A \subseteq\{1,2, \ldots, n\}$ be an orbit under the natural action of $H$ on $\{1,2, \ldots, n\}$. Then the elements of $A$ may be arranged into a sequence $\left(x_{0}, x_{1}, \ldots, x_{2 k-1}\right)$ such that $\pi\left(x_{2 j}\right)=x_{2 j+1}$ and $\rho\left(x_{2 j+1}\right)=x_{(2 j+2) \bmod 2 k}$ for $0 \leq j<k$. In particular, A has even order. 
Proof. Let $x_{0}$ be an arbitrary element of $A$. For each integer $j \in \mathbb{Z}$, we define

$$
x_{2 j}=(\rho \pi)^{j}\left(x_{0}\right), \quad x_{2 j+1}=\left[\pi(\rho \pi)^{j}\right]\left(x_{0}\right) .
$$

Then we have $x_{2 j+1}=\pi\left(x_{2 j}\right)$, and $x_{2 j+2}=(\rho \pi)\left(x_{2 j}\right)=\rho\left(x_{2 j+1}\right)$. We note that each $x_{i}$ is an element of $\{1,2, \ldots, n\}$. Thus there exists a repeated value in the sequence $\left(x_{0}, x_{1}, x_{2}, \ldots\right)$. Let $m$ be the minimum positive index such that $x_{m}$ is an element of $\left\{x_{0}, x_{1}, \ldots, x_{m-1}\right\}$. In particular suppose that $x_{m}=x_{l}$, where $l \in\{0,1, \ldots, m-1\}$.

We claim that $m$ and $l$ have the same parity. If $l$ is even, say $l=2 i$, then for an arbitrary nonnegative integer $j$

$$
x_{l+2 j+1}=\left[\pi(\rho \pi)^{i+j}\right]\left(x_{0}\right)=\left[\pi(\rho \pi)^{j}(\rho \pi)^{i}\right]\left(x_{0}\right)=\left[\pi(\rho \pi)^{j}\right]\left(x_{l}\right) .
$$

And if $l=2 i+1$,

$$
x_{l+2 j+1}=(\rho \pi)^{i+j+1}\left(x_{0}\right)=\left[\rho(\pi \rho)^{j} \pi(\rho \pi)^{i}\right]\left(x_{0}\right)=\left[\rho(\pi \rho)^{j}\right]\left(x_{l}\right) .
$$

But regardless of the parity of $l$, we see that $x_{l+2 j+1} \neq x_{l}$, by 2.4.7. Thus we have our claim, because $x_{m}=x_{l}$. Assume that $m=l+2 k$, where $k$ is a positive integer. Since $m-1$ and $l-1$ have the same parity, $x_{m}=\pi\left(x_{m-1}\right)$ and $x_{l}=\pi\left(x_{l-1}\right)$, or $x_{m}=\rho\left(x_{m-1}\right)$ and $x_{l}=\rho\left(x_{l-1}\right)$. Whichever the case, $x_{m-1}=x_{l-1}$, because $\pi$ and $\rho$ are injective. It follows that $l=0$, because of the minimum condition that we imposed on $m$. Hence $m=2 k$.

Now for an arbitrary integer $j$, we have

$$
x_{2 j+2 k}=(\rho \pi)^{j+k}\left(x_{0}\right)=(\rho \pi)^{j}\left(x_{2 k}\right)=(\rho \pi)^{j}\left(x_{0}\right)=x_{2 j} .
$$


Therefore,

$$
x_{(2 j+1)+2 k}=x_{2(j+k)+1}=\pi\left(x_{2 j+2 k}\right)=\pi\left(x_{2 j}\right)=x_{2 j+1} .
$$

Hence the the function $i \mapsto x_{i}$, defined on $\mathbb{Z}$, has period $2 k$.

We observe that $A=\left\{\varphi\left(x_{0}\right) \mid \varphi \in H\right\}$. Thus $\left\{x_{i} \mid i \in \mathbb{Z}\right\} \subseteq A$. We complete the proof by demonstrating the reverse inclusion. Let $\varphi$ be an element of $H$. Then for some nonnegative integer $t$, there exist elements $\psi_{s} \in\left\{\pi, \pi^{-1}, \rho, \rho^{-1}\right\}, 1 \leq s \leq t$, such that $\varphi=\psi_{1} \psi_{2} \psi_{3} \cdots \psi_{t}$. (See [3, p.62].) However, $\pi^{-1}=\pi$, or equivalently $\pi^{2}=\mathrm{id}$, because $\pi$ is a product of disjoint transpositions. Likewise, $\rho^{-1}=\rho$. Thus by canceling successive factors of $\psi_{1} \psi_{2} \psi_{3} \cdots \psi_{t}$ as long as possible, we obtain

$$
\varphi \in\left\{(\rho \pi)^{j}, \pi(\rho \pi)^{j},(\pi \rho)^{j}, \rho(\pi \rho)^{j}\right\}
$$

for some nonnegative integer $j$. We have $(\rho \pi)^{j}\left(x_{0}\right)=x_{2 j}$, and $\left[\pi(\rho \pi)^{j}\right]\left(x_{0}\right)=x_{2 j+1}$. Furthermore, we observe

$$
\begin{aligned}
(\pi \rho)^{j}\left(x_{0}\right) & =\left[\left(\rho^{-1} \pi^{-1}\right)^{-j}\right]\left(x_{0}\right)=\left[(\rho \pi)^{-j}\right]\left(x_{0}\right)=x_{-2 j}, \\
{\left[\rho(\pi \rho)^{j}\right]\left(x_{0}\right) } & =\left[\pi(\pi \rho)^{j+1}\right]\left(x_{0}\right)=\pi\left(x_{-2(j+1)}\right)=x_{-2(j+1)+1} .
\end{aligned}
$$

Therefore $\varphi\left(x_{0}\right) \in\left\{x_{i} \mid i \in \mathbb{Z}\right\}$. We conclude that $A \subseteq\left\{x_{i} \mid i \in \mathbb{Z}\right\}$, as desired.

Proposition 2.4.9. Let $n, \pi$, and $\rho$ be as in 2.4.7. Then there exists $\varphi \in S_{n}$, also a product of $n / 2$ disjoint transpositions, commuting with $\pi$ and $\rho$.

Proof. Let $H$ be the subgroup of $S_{n}$ generated by $\pi$ and $\rho$. Let $A$ be an arbitrary orbit under the natural action of the subgroup $H$ on $\{1,2, \ldots, n\}$. Then each of the 
elements $\pi$ and $\rho$ maps $A$ to itself, bijectively. Let $\pi_{A}$ and $\rho_{A}$ denote the restrictions of $\pi$ and $\rho$ to $A$, respectively. Let $\left(x_{0}, x_{1}, x_{2}, \ldots, x_{2 k-1}\right)$ be an arrangement of the elements of $A$, as in 2.4.8. For $0 \leq i<k$, define

$$
\varphi_{A}\left(x_{i}\right)=x_{(2 k-1)-i}, \quad \varphi_{A}\left(x_{(2 k-1)-i}\right)=x_{i} .
$$

Since $\pi$ is a product of disjoint transpositions, and $\pi\left(x_{2 j}\right)=x_{2 j+1}$ for $0 \leq j<k$, by design, we see that

$$
\pi_{A}=\left(x_{0} x_{1}\right)\left(x_{2} x_{3}\right)\left(x_{4} x_{5}\right) \cdots\left(x_{2 k-2} x_{2 k-1}\right)
$$

Applying $\varphi_{A}$ to the elements $x_{i}$ within the respective transpositions here, we reverse the sequence of indices and obtain the product

$$
\left(x_{2 k-1} x_{2 k-2}\right)\left(x_{2 k-3} x_{2 k-4}\right)\left(x_{2 k-5} x_{2 k-6}\right) \cdots\left(x_{1} x_{0}\right)=\pi_{A} \text {. }
$$

Hence $\varphi_{A}$ induces a cycle map on $\pi_{A}$. Furthermore, defining $\varphi=\prod_{A} \varphi_{A}$, we realize that $\varphi$ induces a cycle map on $\prod_{A} \pi_{A}=\pi$. Therefore $\varphi$ and $\pi$ are commuting elements, by 2.2 .2 .

Now, since $\rho$ is a product of disjoint transpositions, and $\rho\left(x_{2 j+1}\right)=x_{(2 j+2) \bmod 2 k}$, we have

$$
\rho_{A}=\left(x_{1} x_{2}\right)\left(x_{3} x_{4}\right)\left(x_{5} x_{6}\right) \cdots\left(x_{2 k-3} x_{2 k-2}\right)\left(x_{2 k-1} x_{0}\right) .
$$

Applying $\varphi_{A}$ to the respective $x_{i}$ here yields

$$
\left(x_{2 k-2} x_{2 k-3}\right)\left(x_{2 k-4} x_{2 k-5}\right)\left(x_{2 k-6} x_{2 k-7}\right) \cdots\left(x_{2} x_{1}\right)\left(x_{0} x_{2 k-1}\right)=\rho_{A} \text {. }
$$


Therefore, we see that $\varphi$ induces a cycle map on $\rho$, so $\varphi$ and $\rho$ are commuting elements, by 2.2.2. And $\varphi$ is obviously a product of disjoint transpositions, fixing no element of $\{1,2, \ldots, n\}$. Thus the proof is complete.

We have now realized our goal of a more constructive route to Proposition 2.4.6. But furthermore, Proposition 2.4.9 yields a new proof of a result of [1, p.139].

Theorem 2.4.10. Suppose that $n$ is even, $n>4$. Let $X$ be the set of all products of $n / 2$ disjoint transpositions in $S_{n}$. Then the commuting graph $\Delta_{X}$ has diameter 2.

Proof. The diameter of $\Delta_{X}$ is $\leq 2$ by 2.4.9. Define

$$
\begin{aligned}
\pi & =(12)(34)(56) \cdots(n-1 n) \\
\rho & =(23)(45)(67) \cdots(n-2 n-1)(1 n),
\end{aligned}
$$

a particular pair of elements of $X$. We observe that $(\rho \pi)(1)=\rho(2)=3$, while $(\pi \rho)(1)=\pi(n)=n-1$. Therefore $(\rho \pi)(1) \neq(\pi \rho)(1)$, because $n>4$. Hence $\pi$ and $\rho$ are not commuting elements. We conclude that the diameter of $\Delta_{X}$ is $>1$.

We remark that in the terminology of [1], $\Delta_{X}$ is referred to as a commuting involution graph. 


\subsection{An upper bound on the diameter of $\Delta_{S_{n}^{*}}$ for composite $n$ and $n-1$}

Theorem 2.5.1. Suppose that each of $n$ and $n-1$ is a composite number. Then the diameter $\Delta_{S_{n}^{*}}$ is $\leq 5$.

We remark that $n \geq 9$ here, implicitly. We obtain the theorem through two propositions, that shall accompany Theorem 2.3.1.

Proposition 2.5.2. Suppose that $n>4$, and $l \in\{n-1, n\}$ is a composite number. Let $\pi \in R_{n}$ and $\gamma \in C_{n}^{l}$. Then the distance between $\pi$ and $\gamma$ in $\Delta_{R_{n} \cup C_{n}^{l}}$ is $\leq 5$.

Proof. By 2.3.3, there exists a cycle $\delta \in R_{n}$ of length $\leq n / 2$ that commutes with $\pi$. We have $|F(\delta)| \geq n / 2$, thus $|F(\delta)| \geq 3$ because $n>4$. Choose $a, b \in F(\delta)$, and define $\tau=(a b) \in R_{n}$. Then $\tau$ is disjoint from $\delta$; hence $\tau$ and $\delta$ are commuting elements, by 2.2 .9 .

Now assume that $\gamma=\left(\begin{array}{lllll}c_{1} & c_{2} & c_{3} & \cdots & c_{l}\end{array}\right)$. Since $l$ is composite, there exists a positive integer $i \in(1, l)$ that divides $l$. We observe that $\gamma$ commutes with $\gamma^{i}$, because a group element commutes with each of its powers. In decomposed form, we have

$$
\gamma^{i}=\prod_{j=1}^{i}\left(a_{j} a_{j+i} a_{j+2 i} \cdots a_{j+[(l / i)-1] i}\right) .
$$

In particular, $\gamma^{i}$ is a product of $i \geq 2$ disjoint cycles, each of length $l / i \geq 2$. Hence $\gamma^{i} \in R_{n}$. Moreover since $\tau$ is a cycle of length $\leq i$, there exists an element $\rho \in R_{n}$ 
commuting with $\gamma^{i}$ and $\tau$, by 2.4.3. Therefore, $\left(\pi, \delta, \tau, \rho, \gamma^{i}, \gamma\right)$ is a commuting path in $\Delta_{R_{n} \cup C_{n}^{l}}$. The proposition follows.

Proposition 2.5.3. Let $l$ and $m$ be elements of the set $\{n-1, n\}$. Assume that $l \leq m$, and that each of $l$ and $m$ is a composite number. Let $\gamma \in C_{n}^{l}$, and $\delta \in C_{n}^{m}$. Then the distance between $\gamma$ and $\delta$ in $\Delta_{R_{n} \cup C_{n}^{l} \cup C_{n}^{m}}$ is $\leq 5$.

Proof. Let $D_{l} \subseteq\{2,3, \ldots, l-1\}$ be the set of all proper nontrivial divisors of $l$. Since $l$ is composite, $D_{l}$ is nonemtpy. Let $i=\max \left(D_{l}\right)$. Since $i \in D_{l}$, we have $l / i \in D_{l}$ as well. Therefore $l / i \leq i$, because $i$ is maximal; so $\sqrt{l} \leq i$. Analogously, we let $D_{m} \subseteq\{2,3, \ldots, m-1\}$ be the collection of all proper nontrivial divisors of $m$, nonempty because $m$ is composite, and we let $j=\max \left(D_{m}\right)$. We then note that $m / j \in D_{m}$, and deduce that $\sqrt{m} \leq j$. Moreover we have $\sqrt{l} \leq j$, because $l \leq m$. Hence $l \leq i j$, and so $l / i \leq j$.

Now assume that

$$
\gamma=\left(a_{1} a_{2} a_{3} \cdots a_{l}\right), \quad \delta=\left(b_{1} b_{2} b_{3} \cdots b_{m}\right) .
$$

Then we have, in decomposed form,

$$
\begin{aligned}
& \gamma^{i}=\prod_{k=1}^{i}\left(a_{k} a_{k+i} a_{k+2 i} \cdots a_{k+[(l / i)-1] i}\right) \\
& \delta^{j}=\prod_{k=1}^{j}\left(b_{k} b_{k+j} b_{k+2 j} \cdots b_{k+[(m / j)-1] j}\right) .
\end{aligned}
$$

We observe that $\gamma^{i}$ consists of $i$ nontrivial cycles, each of length $l / i$, and $\delta^{j}$ consists of $j$ nontrivial cycles, each of length $m / j$. So obviously, $\gamma^{i}, \delta^{j} \in R_{n}$. Let $\sigma$ be any 
nontrivial cycle in the decomposition of $\gamma^{i}$. Then $\sigma \in R_{n}$, because $\gamma^{i}$ has multiple nontrivial cycles. By 2.2.3, $\sigma$ commutes with $\gamma^{i}$. Furthermore since $l / i \leq j$, there exists an element $\rho \in R_{n}$ that commutes with $\sigma$ and $\delta^{j}$, by 2.4.3. Hence $\left(\gamma, \gamma^{i}, \sigma, \rho, \delta^{j}, \delta\right)$ is a commuting path in $\Delta_{R_{n} \cup C_{n}^{l} \cup C_{n}^{m}}$, because $\gamma$ and $\delta$ commute with $\gamma^{i}$ and $\delta^{j}$, respectively. Thus we have the proposition.

We finish the section with an argument for Theorem 2.5.1.

Proof. As noted earlier, we have $n \geq 9$, because $n$ and $n-1$ are composite numbers. The theorem is realized immediately by combining the results of Theorem 2.3.1, and Propositions 2.5.2 and 2.5.3.

\subsection{The existence of elements at distance 5 in $\Delta_{S_{n}^{*}}$}

We exhibit two pairs of elements at distance $\geq 5$ in the commuting graph $\Delta_{S_{n}^{*}}$. In each of our constructions, we shall require the following standard result.

Lemma 2.6.1. Let $G$ be a group. Suppose that $g \in G$ has finite order $i$. Then for all integers $j,\left\langle g^{j}\right\rangle=\left\langle g^{\operatorname{gcd}(i, j)}\right\rangle$.

Proof. We observe that $\operatorname{gcd}(i, j)$ divides $j$; suppose $j=k \cdot \operatorname{gcd}(i, j)$, where $k \in \mathbb{Z}$. Then we have $g^{j}=\left(g^{\operatorname{gcd}(i, j)}\right)^{k} \in\left\langle g^{\operatorname{gcd}(i, j)}\right\rangle$. Thus $\left\langle g^{j}\right\rangle \subseteq\left\langle g^{\operatorname{gcd}(i, j)}\right\rangle$.

Now, by a well-known result of number theory, there exist integers $x$ and $y$ such that $i x+j y=\operatorname{gcd}(i, j)$. (See [4, p.11].) Therefore, $g^{\operatorname{gcd}(i, j)}=\left(g^{i}\right)^{x}\left(g^{j}\right)^{y}=\left(g^{j}\right)^{y}$, because $g$ has order $i$. Hence $g^{\operatorname{gcd}(i, j)} \in\left\langle g^{j}\right\rangle$, and so $\left\langle g^{\operatorname{gcd}(i, j)}\right\rangle \subseteq\left\langle g^{j}\right\rangle$. 
Proposition 2.6.2. Suppose that $n$ is a positive integer, $n \geq 3$. Let

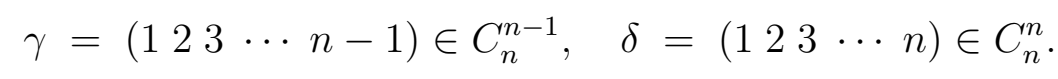

Then the distance between $\gamma$ and $\delta$ in $\Delta_{S_{n}^{*}}$ is at least 5 .

Proof. We note that $\gamma$ and $\delta$ are in fact elements of $S_{n}^{*}$, because $n \geq 3$. Suppose that $d_{\Delta_{S_{n}^{*}}}(\gamma, \delta) \leq 4$. In particular, assume that $(\gamma, \varphi, \chi, \psi, \delta)$ is a commuting path in $\Delta_{S_{n}^{*}}$. Since $\varphi$ and $\psi$ commute with $\gamma$ and $\delta$, respectively, there exist positive integers $s$ and $t$ such that $\varphi=\gamma^{s}$ and $\psi=\delta^{t}$, by 2.2.4. Let $u=\operatorname{gcd}(s, n-1)$ and $v=\operatorname{gcd}(t, n)$; and note that $u$ and $v$ are proper divisors of $n-1$ and $n$, respectively, because $\varphi$ and $\psi$ are nontrivial elements. Let $\pi=\gamma^{u}, \rho=\delta^{v}$, and $H=\langle\pi, \rho\rangle$. By 2.6.1, we have $\langle\varphi\rangle=\langle\pi\rangle$ and $\langle\psi\rangle=\langle\rho\rangle$. Hence each subgroup of $S_{n}$ that contains $\varphi$ and $\psi$ will also contain $\pi$ and $\rho$, and vice-versa. Therefore, $H=\langle\varphi, \psi\rangle$.

We observe that precisely one of the integers $n-1$ and $n$ is divisible by 2 ; so $u+v<(n-1) / 2+n / 2$. Thus $u+v$, itself an integer, must be $\leq n-1$. Let $m=\min (u, v)$, and let $a \in\{n-m, n-m+1, n-m+2, \ldots, n-1\}$. We observe that $n-1$ is strictly less than $a+u$ and $a+v$, but $a+u+v \leq 2(n-1)$. Therefore,

$$
\begin{aligned}
(\rho \pi)(a) & =\rho[a+u-(n-1)]=a+u+v-(n-1), \\
(\pi \rho)(a+1) & =\pi[(a+1)+v-n]=\pi[a+v-(n-1)]=a+u+v-(n-1) .
\end{aligned}
$$

Thus $(\rho \pi)(a)=(\pi \rho)(a+1)$, and so $\left(\rho^{-1} \pi^{-1} \rho \pi\right)(a)=a+1$. Hence $a+1 \in[a]_{H}$, because $\rho^{-1} \pi^{-1} \rho \pi \in H$. Moreover $[a]_{H}=[a+1]_{H}$, by 2.2.11. Therefore, we conclude 
that

$$
[n-m]_{H}=[n-m+1]_{H}=\cdots=[n-1]_{H}=[n]_{H}
$$

Suppose that $b \in\{1,2, \ldots, n-m-1\}$. We observe that $i=0$ is a solution to $b+i m<n-m$; thus there exists a maximum nonnegative integer $i$ for which the inequality holds. For this $i$, we have

$$
b+(i+1) m \in\{n-m, n-m+1, n-m+2, \ldots, n-1\} .
$$

We observe that if $m=u$, then $\pi^{i+1}(b)=b+(i+1) m$. And if $m=v$, then $\rho^{i+1}(b)=b+(i+1) m$. Either way, we have $[b]_{H}=[b+(i+1) m]_{H}$, because each of $\pi^{i+1}$ and $\rho^{i+1}$ is an element of $H$. Together with (2.6.1), this implies that

$$
[1]_{H}=[2]_{H}=\cdots=[n-1]_{H}=[n]_{H} .
$$

Hence $[n]_{H}=\{1,2, \ldots, n\}$.

Define $K=\langle\varphi, \chi, \psi\rangle$. Then $K$ may be explicitly described as the set of all products of the form $\eta_{1} \eta_{2} \cdots \eta_{w}$, where $w$ is a positive integer, and each $\eta_{j}$ is an element of $\left\{\varphi, \varphi^{-1}, \chi, \chi^{-1}, \psi, \psi^{-1}\right\}$. (See [3, p.62].) We recall that $\chi$ sits between $\varphi$ and $\psi$ in our commuting path; hence $\chi$ commutes with $\varphi$ and $\psi$. Therefore $\chi$ commutes with $\varphi^{-1}$ and $\psi^{-1}$ as well. And of course $\chi$ commutes with itself and its inverse. Thus $\chi$ commutes with all products $\eta_{1} \eta_{2} \cdots \eta_{w}$. In other words, $\chi$ is a member of the center of $K$.

Since $\varphi \in\langle\gamma\rangle \backslash\{\mathrm{id}\}$, we see that $F(\varphi)=\{n\}$. Therefore by 2.2.6, $n \in F(\chi)$, because $\varphi$ and $\chi$ are commuting elements. Moreover since $\chi$ is an element of the 
center of $K$, we have $[n]_{K} \subseteq F(\chi)$, by 2.2.12. But $H$ is a subgroup of $K$, because $H=\langle\varphi, \psi\rangle$ and $\varphi, \psi \in K$. Thus $[n]_{H} \subseteq[n]_{K}$, and so $[n]_{K}=\{1,2, \ldots, n\}$. We conclude that $F(\chi)=\{1,2, \ldots, n\}$, which implies that $\chi$ is the identity element of $S_{n}$. This is a contradiction. Hence we have the proposition.

To prove the main result of the current chapter, we must still demonstrate the existence of a pair of elements at distance 5 in $\Delta_{R_{n} \cup C_{n}^{n-1}}$, when $n-1$ is composite. For the remainder of the section, the following setup shall apply.

- Let $n$ be a positive integer such that $n-1$ is a composite number.

- Let $M$ be the maximum proper divisor of $n-1$.

- Define the following elements of $C_{n}^{n-1}$ :

$$
\begin{aligned}
\gamma & =(12 \cdots n-1) \\
\delta & =(12 \cdots M n M+1 M+2 \cdots n-2) .
\end{aligned}
$$

- Let $p$ and $q$ be arbitrary prime divisors of $n-1$, possibly alike.

- Let $r=(n-1) / p, s=(n-1) / q$, and $m=\min (r, s)$.

- Define $H=\left\langle\gamma^{r}, \delta^{s}\right\rangle$.

We note that $n \geq 5, M>1$, and $m>1$, because $n-1$ is composite. We also point out that $r$ and $s$ are proper divisors of $n-1$, hence $r, s \leq M$. 
Lemma 2.6.3. Suppose that $a$ is an integer such that $M+1-m \leq a \leq M-2$. Then $[a]_{H}=[a+2]_{H}$

Proof. First assume that $r \leq s$. We observe that

$$
M+1 \leq a+r \leq 2 M-2 \leq n-3
$$

Let $i$ be the maximum positive integer such that $M+1 \leq a+i r \leq n-2$. Then since $r \leq s$, we have $n-2<a+i r+s \leq(n-2)+M$. Therefore

$$
\left(\delta^{s} \gamma^{i r}\right)(a)=\delta^{s}(a+i r)=a+i r+s-(n-2)
$$

But we have $M+1 \leq a+s \leq n-3$ as well, so

$$
\left(\gamma^{i r} \delta^{s}\right)(a+2)=\gamma^{i r}(a+s+1)=a+i r+s+1-(n-1) .
$$

Hence $\left(\delta^{s} \gamma^{i r}\right)(a)=\left(\gamma^{i r} \delta^{s}\right)(a+2)$, and thus $\left(\delta^{-s} \gamma^{-i r} \delta^{s} \gamma^{i r}\right)(a)=a+2$. It follows that $[a]_{H}=[a+2]_{H}$, because $\delta^{-s} \gamma^{-i r} \delta^{s} \gamma^{i r} \in H$.

Now let us assume that $s<r$. Let $j$ be the maximum positive integer such that $M+1 \leq a+j s \leq n-3$. Then $n-2<a+r+j s \leq(n-3)+M$, because $s$ is strictly less than $r$. Thus

$$
\left(\gamma^{r} \delta^{j s}\right)(a+2)=\gamma^{r}(a+j s+1)=a+r+j s+1-(n-1) .
$$

But on the other hand,

$$
\left(\delta^{j s} \gamma^{r}\right)(a)=\delta^{j s}(a+r)=a+r+j s-(n-2)
$$

Thus $\left(\gamma^{r} \delta^{j s}\right)(a+2)=\left(\delta^{j s} \gamma^{r}\right)(a)$. So once again, $[a]_{H}=[a+2]_{H}$. 
Lemma 2.6.4. Suppose that $a$ and $b$ are integers such that $M-m+1 \leq a, b \leq M$. Assume that $a$ and $b$ have opposite parity. Then $[a]_{H} \cup[b]_{H}=\{1,2, \ldots, n\}$.

Proof. Let $i$ and $j$ be the odd and even elements of the set $\{m-1, m\}$, respectively. Define

$$
\begin{aligned}
C & =\{M-m+1, M-m+3, M-m+5, \ldots, M-m+i\}, \\
D & =\{M-m+2, M-m+4, M-m+6, \ldots, M-m+j\} .
\end{aligned}
$$

We observe that $C \cup D=\{M-m+1, M-m+2, \ldots, M-1, M\}$, so $a, b \in C \cup D$. Also, either the elements of $C$ are strictly even and those of $D$ are strictly odd, or vice versa. Thus one of the elements $a$ and $b$ is a member of $C$, and the other is a member of $D$. But by 2.6.3,

$$
\begin{aligned}
{[M-m+1]_{H} } & =[M-m+3]_{H}=[M-m+5]_{H}=\cdots=[M-m+i]_{H}, \\
{[M-m+2]_{H} } & =[M-m+4]_{H}=[M-m+6]_{H}=\cdots=[M-m+j]_{H} .
\end{aligned}
$$

Therefore $C \cup D \subseteq[a]_{H} \cup[b]_{H}$.

Suppose $1 \leq x \leq M-m$. Let $k$ be the maximum nonnegative integer such that $x+k m \leq M-m$, and let $y=x+(k+1) m$. Then $y \in C \cup D$, because $C \cup D$ consists of $m$ consecutive integers. We observe that $\gamma^{(k+1) m}(x)=\delta^{(k+1) m}(x)=y$. Also, if $m=r$ then $\gamma^{(k+1) m}=\left(\gamma^{r}\right)^{k+1} \in H$, and if $m=s$ then $\delta^{(k+1) m}=\left(\delta^{s}\right)^{k+1} \in H$. Therefore, $[x]_{H}=[y]_{H}$. But $y \in[a]_{H}$ or $y \in[b]_{H}$, because $y \in C \cup D$. Hence $x \in[a]_{H}$ or $x \in[b]_{H}$. In other words, $x \in[a]_{H} \cup[b]_{H}$. 
Now assume that $M+1 \leq z \leq n-1$. Let $l$ be the maximum nonnegative integer such that $z-l r \geq M+1$. Then $z-(l+1) r \in\{1,2, \ldots, M\}$, since $r \leq M$. And we have $\gamma^{-(l+1) r}(z)=z-(l+1) r$. Therefore $[z]_{H}=[z-(l+1) r]_{H}$, because $\gamma^{-(l+1) r}=\left(\gamma^{r}\right)^{-(l+1)} \in H$. But we have already shown that $\{1,2, \ldots, M\} \subseteq[a]_{H} \cup[b]_{H}$. Thus $z \in[a]_{H} \cup[b]_{H}$.

Finally, we observe that $\delta^{-s}(n)=M-s+1$. Hence $[n]_{H}=[M-s+1]_{H}$. Therefore $n \in[a]_{H} \cup[b]_{H}$, because $M-s+1 \in\{1,2, \ldots, M-1\} \subseteq[a]_{H} \cup[b]_{H}$.

Proposition 2.6.5. Suppose $p=2$ or $q=2$, but $p \neq q$. Then

$$
[n-1]_{H} \cup[n]_{H}=\{1,2, \ldots, n\}
$$

Proof. Since $2 \in\{p, q\}$, and $p$ and $q$ are divisors of $n-1$, we realize that $n-1$ is even. Therefore $M=(n-1) / 2$.

Assume that $p=2$. Then we have $r=M$ and $s=m$; thus $\gamma^{r}(n-1)=M$ and $\delta^{-s}(n)=M-m+1$. Therefore $[n-1]_{H}=[M]_{H}$, and $[n]_{H}=[M-m+1]_{H}$. Since $p \neq q, q$ is an odd prime. Hence $s$ is even, because $n-1$ is even, and so $M$ and $M-m+1$ have opposite parity, because $s=m$. Thus by 2.6 .4 , we have $[M]_{H} \cup[M-m+1]_{H}=\{1,2, \ldots, n\}$, and therefore $[n-1]_{H} \cup[n]_{H}=\{1,2, \ldots, n\}$.

Now suppose that $q=2$. Then by analogy to the above case, $r=m$ and $s=M$, and $r$ is even. We observe that the set $\{M-m+1, M-m+2, \ldots, M\}$ consists of $r$ consecutive integers. Therefore the set includes elements $a$ and $b$ such that $a \equiv 0(\bmod r)$ and $b \equiv 1(\bmod r)$. Let $i$ and $j$ be integers such that $a=i r$ and 
$b=j r+1$. Then $\gamma^{i r}(n-1)=a$, and $\left(\gamma^{j r} \delta^{-s}\right)(n)=\gamma^{j r}(1)=b$, because $s=M$. Hence $[n-1]_{H}=[a]_{H}$, and $[n]_{H}=[b]_{H}$. But $a$ and $b$ have opposite parity, because $r$ is even. Thus by 2.6.4 once again, we have $[n-1]_{H} \cup[n]_{H}=\{1,2, \ldots, n\}$.

We point out that $[n-1]_{H} \neq[n]_{H}$ is a possibility under the hypotheses of 2.6.5. For example if $n-1=6$, then

$$
\gamma=(123456), \quad \delta=(123745)
$$

If $p=2$ and $q=3$, we have $r=3$ and $s=2$. Therefore $\gamma^{r}=(14)(25)(36)$, and

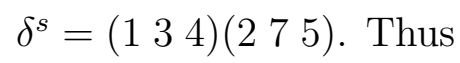

$$
[6]_{H}=\{1,3,4,6\}, \quad[7]_{H}=\{2,5,7\} .
$$

Definition 2.6.6. If the natural action of $H$ on $\{1,2, \ldots, n\}$ has precisely one orbit, then we shall say that $H$ is transitive.

Proposition 2.6.7. If $p=q=2$, then $H$ is transitive.

Proof. We observe that $r=s=M=(n-1) / 2$. Thus for $1 \leq a \leq M-1$, we have

$$
\left(\delta^{-s} \gamma^{r}\right)(a)=\left(\delta^{-M} \gamma^{M}\right)(a)=\delta^{-M}(a+M)=a+1
$$

Therefore $[a]_{H}=[a+1]_{H}$, and furthermore, $[1]_{H}=[2]_{H}=\cdots=[M]_{H}$.

Now, for $M+2 \leq a \leq n-1$,

$$
\left(\delta^{s} \gamma^{-r}\right)(a)=\delta^{M}(a-M)=a-1
$$

Hence $[a]_{H}=[a-1]_{H}$, and so $[M+1]_{H}=[M+2]_{H}=\cdots=[n-1]_{H}$. 
Finally, we notice that $\gamma^{r}(n-1)=M$, and $\delta^{-s}(n)=1$. Therefore we have $[n-1]_{H}=[M]_{H}$, and $[n]_{H}=[1]_{H}$. So we conclude that

$$
[1]_{H}=[2]_{H}=\cdots=[n-1]_{H}=[n]_{H}
$$

Lemma 2.6.8. If each of $r$ and $s$ is $\leq n-M-3$, then $H$ is transitive.

Proof. Let $a=M-m+1$. Since $m \geq 2$, the set $\{M-m+1, M-m+2, \ldots, M\}$ contains at least two elements; thus $\{a, a+1\}$ is a subset. We point out that each of $a+r$ and $a+s$ is $\geq M+1$, but that

$$
a+r+s=M+\max (r, s)+1 \leq M+(n-M-3)+1=n-2 .
$$

Therefore,

$$
\begin{aligned}
\left(\delta^{s} \gamma^{r}\right)(a) & =\delta^{s}(a+r)=a+r+s \\
\left(\gamma^{r} \delta^{s}\right)(a+1) & =\gamma^{r}(a+s)=a+r+s .
\end{aligned}
$$

Hence $\left(\delta^{-s} \gamma^{-r} \delta^{s} \gamma^{r}\right)(a)=a+1$, and so $[a]_{H}=[a+1]_{H}$. But by 2.6.4, we have $[a]_{H} \cup[a+1]_{H}=\{1,2, \ldots, n\}$. Thus the lemma follows.

Proposition 2.6.9. If $p \neq 2$ and $q \neq 2$, then $H$ is transitive.

Proof. We observe that $n-1$, being divisible by an odd prime, is not a power of 2 . In the case of $n-1=6$ and $p=q=3, \gamma$ and $\delta$ are as in equation (2.6.4), and $r=s=2$. Therefore $\gamma^{r}=\left(\begin{array}{lll}1 & 3 & 5\end{array}\right)(246)$, and $\delta^{s}=\left(\begin{array}{lll}1 & 3 & 4\end{array}\right)(275)$. Thus we obviously have

$$
[1]_{H}=[3]_{H}=[5]_{H}, \quad[2]_{H}=[4]_{H}=[6]_{H} ; \quad[1]_{H}=[3]_{H}=[4]_{H}, \quad[2]_{H}=[7]_{H}=[5]_{H}
$$


Hence we see that $[1]_{H}=[2]_{H}=\cdots=[7]_{H}$, and so $H$ is transitive.

For $n-1=9$, we have $p=q=r=s=M=3$. And for $n-1=10$, $p=q=M=5$ and $r=s=2$. But in either case, each of $r$ and $s$ is $<n-M-3$. Therefore $H$ is transitive, by 2.6.8.

Now assume that $n-1 \geq 12$. Since $p$ and $q$ are both odd primes, each of $r$ and $s$ is $\leq(n-1) / 3$. Therefore

$$
M+\max (r, s) \leq \frac{n-1}{2}+\frac{n-1}{3}=n-\frac{n+5}{6} \leq n-3 .
$$

Thus by 2.6.8, $H$ is transitive once again.

In the proof of the culminating result of the current section, as follows, the prime numbers $p$ and $q$ that have been under consideration, and thus the group $H$, shall arise. In stating the proposition, we keep our assumptions that $n-1$ is composite, and $M$ is the maximum proper divisor of $n-1$. The definitions of $\gamma$ and $\delta$, as in (2.6.2) and (2.6.3), remain as well. Our argument here revisits many of the techniques that we applied in the proof of 2.6.2.

Proposition 2.6.10. The distance between $\gamma$ and $\delta$ in $\Delta_{S_{n}^{*}}$ is at least 5 .

Proof. Suppose that the distance between $\gamma$ and $\delta$ in $\Delta_{S_{n}^{*}}$ is $\leq 4$. In particular, assume that $(\gamma, \varphi, \chi, \psi, \delta)$ is a commuting path in $\Delta_{S_{n}^{*}}$. Since $\varphi$ and $\psi$ commute with $\gamma$ and $\delta$, respectively, there exist positive integers $t$ and $u$ such that $\varphi=\gamma^{t}$ and $\psi=\delta^{u}$, by 2.2.4. We observe that $\gamma^{\operatorname{gcd}(t, n-1)} \in\langle\varphi\rangle$, and $\delta^{\operatorname{gcd}(u, n-1)} \in\langle\psi\rangle$, by 2.6.1. Also, $\operatorname{gcd}(t, n-1)$ and $\operatorname{gcd}(u, n-1)$ are proper divisors of $n-1$, because $\varphi$ and $\psi$ are 
nontrivial elements. Suppose that $n-1=p \cdot j \cdot \operatorname{gcd}(t, n-1)=q \cdot k \cdot \operatorname{gcd}(u, n-1)$, where $p$ and $q$ are prime numbers, and $j$ and $k$ are positive integers. Since $\gamma^{\operatorname{gcd}(t, n-1)} \in\langle\varphi\rangle$, we have $\left(\gamma^{\operatorname{gcd}(t, n-1)}\right)^{j}=\gamma^{(n-1) / p} \in\langle\varphi\rangle$ as well. Similarly, $\delta^{(n-1) / q} \in\langle\psi\rangle$. And we note that $\gamma^{(n-1) / p}$ and $\delta^{(n-1) / q}$ are nontrivial elements, because each of $(n-1) / p$ and $(n-1) / q$ is strictly less than $n-1$.

Now given the structure of our commuting path, we see that $\chi$ commutes with $\varphi$ and $\psi$. So furthermore, $\chi$ commutes with each element of $\langle\varphi\rangle$, and each of $\langle\psi\rangle$. Hence $\chi$ commutes with $\gamma^{(n-1) / p}$ and $\delta^{(n-1) / q}$.

Let us define

$$
K=\left\langle\gamma^{(n-1) / p}, \chi, \delta^{(n-1) / q}\right\rangle
$$

We observe that $\chi$ is a member of the center of $K$, as in the proof of 2.6.2. Also, by 2.2.6, $n-1$ and $n$ are fixed by $\chi$, because we obviously have $F\left(\gamma^{(n-1) / p}\right)=\{n\}$ and $F\left(\delta^{(n-1) / q}\right)=\{n-1\}$. Moreover we have $[n-1]_{K} \cup[n]_{K} \subseteq F(\chi)$, by 2.2.12. But letting $H=\left\langle\gamma^{(n-1) / p}, \delta^{(n-1) / q}\right\rangle$, we realize that $[n-1]_{H} \cup[n]_{H}=\{1,2, \ldots, n\}$, in view of Propositions 2.6.5, 2.6.7, and 2.6.9. Hence $[n-1]_{K} \cup[n]_{K}=\{1,2, \ldots, n\}$, because $H$ is a subgroup of $K$. We conclude that $F(\chi)=\{1,2, \ldots, n\}$. In other words, $\chi$ is the identity element of $S_{n}$. This is a contradiction, so the proof is complete. 


\subsection{Proof of main result}

In order to realize the main result of the current chapter, we prove one further proposition.

Proposition 2.7.1. Suppose that $p \in\{n-1, n\}$ is a prime number. Let $\gamma$ be an element of $C_{n}^{p}$. Then $\Delta_{\langle\gamma\rangle^{*}}$ is a connected component of $\Delta_{S_{n}^{*}}$. Furthermore, the diameter of $\Delta_{\langle\gamma\rangle^{*}}$ is 0 or 1 , according as $p=2$ or $p>2$.

Proof. We observe that $\langle\gamma\rangle$ is an abelian group, containing $p-1$ nontrivial elements. Thus we see that the diameter of $\Delta_{\langle\gamma\rangle^{*}}$ is equal to 0 if $p=2$, but equal to 1 if $p>2$. Suppose that the connected component of $\gamma$ in $\Delta_{S_{n}^{*}}$ strictly contains $\Delta_{\langle\gamma\rangle^{*}}$. Then there exists a commuting path $(\gamma, \pi, \rho)$ in $\Delta_{S_{n}^{*}}$ such that $\gamma$ and $\rho$ are noncommuting elements. Since $\pi$ commutes with $\gamma$, we have $\pi \in\langle\gamma\rangle$, by 2.2.4. Therefore the order of $\pi$ is a divisor of $p$, the order of $\gamma$, by Lagrange's Theorem. (See [3, p.89].) Thus $\pi$ has order $p$, because $p$ is a prime and $\pi$ is nontrivial. It follows that $\langle\pi\rangle=\langle\gamma\rangle$. Furthermore, we claim that $\pi$ is itself a cycle of length $p$. We observe that if $l$ is a positive integer, and $l \notin\{1, p\}$, then the decomposition of $\pi$ cannot contain a cycle of length $l$, because the order of $\pi$ is not divisible by $l$. Also, the decomposition of $\pi$ cannot have two disjoint cycles of length $p$, since $2 p>n$. Hence we have our claim. Therefore by 2.2.4, $\rho \in\langle\pi\rangle$, because $\rho$ and $\pi$, being adjacent in our commuting path, are commuting elements. Thus $\rho \in\langle\gamma\rangle$. In particular, we conclude that $\rho$ and $\gamma$ are commuting elements, which is a contradiction. Thus we have the proposition. 
We may now give arguments to obtain Theorem 2.1.2.

Proof. We consider the three cases separately.

a. This follows at once from Theorem 2.5.1 and Proposition 2.6.2.

b. First consider the case of $n=3$. We observe that $R_{3}=\emptyset$, and

$$
C_{3}^{3}=\left\{\left(\begin{array}{lll}
1 & 2 & 3
\end{array}\right),\left(\begin{array}{lll}
1 & 3 & 2
\end{array}\right)\right\}=\left\langle\left(\begin{array}{lll}
1 & 2 & 3
\end{array}\right)\right\rangle^{*} .
$$

Thus $\Delta_{R_{3} \cup C_{3}^{3}}$ is a connected component of $\Delta_{S_{3}^{*}}$, of diameter 1 , by 2.7.1. Also, for $\gamma \in C_{3}^{2}, \Delta_{\langle\gamma\rangle^{*}}$ is a component of $\Delta_{S_{n}^{*}}$ of diameter 0 , by 2.7.1.

Regarding the $n=4$ case, we observe that for each $\gamma \in C_{4}^{3}, \Delta_{\langle\gamma\rangle^{*}}$ is a connected component of $\Delta_{S_{4}^{*}}$, of diameter 1 , by 2.7.1. And by 2.4.1, $\Delta_{R_{4} \cup C_{4}^{4}}$ is connected of diameter 3. Thus we see that $\Delta_{R_{4} \cup C_{4}^{4}}$ is a component of $\Delta_{S_{4}^{*}}$, in particular. For $n>4$, we note that $n$ is even, because $n-1$ is a prime. Therefore by 2.4 .1 and 2.7.1, once again, we realize that the connected components of $\Delta_{S_{n}^{*}}$ are $\Delta_{R_{n} \cup C_{n}^{n}}$, of diameter 4 , and $\Delta_{\langle\gamma\rangle^{*}}$ for $\gamma \in C_{n}^{n-1}$, each of diameter 1 .

c. By 2.7.1, $\Delta_{\langle\gamma\rangle^{*}}$ is a connected component of $\Delta_{S_{n}^{*}}$, of diameter 1 , for each $\gamma \in C_{n}^{n}$. We observe that $n-1$ is even and $>2$, because $n$ is a prime and $>3$. Hence $n-1$ is composite. Therefore by $2.3 .4,2.5 .2,2.5 .3$, and $2.6 .10, \Delta_{R_{n} \cup C_{n}^{n-1}}$ is connected of diameter 5 . Thus we see that $\Delta_{R_{n} \cup C_{n}^{n-1}}$ is a component of $\Delta_{S_{n}^{*}}$. 


\section{Chapter 3}

\section{A Diameter Bounding Result and Certain Solvable Groups}

\section{$3.1 \quad$ Summary}

In this brief chapter, we develop an absolute bound for the commuting graphs $\Delta_{G^{*}}$ over a particular class of groups. We require a definition to precisely specify the class.

Definition 3.1.1. Suppose $n$ is an integer, $n \geq 3$. Let $Q_{2^{n}}$ be the group with presentation $\left\langle x, y \mid x^{2^{n-1}}=1, y^{2}=x^{2^{n-2}}, y x=x^{-1} y\right\rangle$. Then $Q_{2^{n}}$ is called the generalized quaternion group of order $2^{n}$.

We remark that each element of $Q_{2^{n}}$ may be reduced to the form $x^{i} y^{j}$, where $i \in\left\{0,1,2, \ldots, 2^{n-1}\right\}$ and $j \in\{0,1\}$. Furthermore we see that if $x^{i_{1}} y^{j_{1}}$ and $x^{i_{2}} y^{j_{2}}$ have this form, then these products represent the same element if and only if $i_{1}=i_{2}$ and $j_{1}=j_{2}$. Thus $Q_{2^{n}}$ does indeed have order $2^{n}$. For more on generalized quaternion groups, we refer the reader to [10, pp.140-141].

The following theorem constitutes the primary result of the chapter. 
Theorem 3.1.2. Suppose that $G$ is a finite group with a nontrivial abelian normal subgroup. Assume that $G$ contains no cyclic or generalized quaternion Sylow subgroups. Then $\Delta_{G^{*}}$ is connected, of diameter $\leq 7$.

However, we take particular interest in a straightforward corollary, settling the question of connectivity for a significant collection of solvable groups.

Corollary 3.1.3. Suppose that $G$ is a nontrivial finite solvable group, with no cyclic or generalized quaternion Sylow subgroups. Then $\Delta_{G^{*}}$ is connected of diameter $\leq 7$.

\subsection{Arguments}

Lemma 3.2.1. Let $G$ be a finite group. Suppose that $N$ and $H$ are nontrivial abelian subgroups of $G$. Furthermore assume that $N$ is normal in $G$, and $H$ is noncyclic. Then there exist nontrivial commuting elements $w \in N^{*}$ and $h \in H^{*}$.

Proof. Let $p$ be a prime dividing the order of $N$, and let $V$ be the set of all elements of $N$ having order $p$, together with the identity element of $N$. We observe that $V$ is nontrivial, by Cauchy's Theorem. (See [4, p.93].) Furthermore $V$ is an abelian group, because $N$ is an abelian group, and a product of commuting elements of order $p$ yields an element of order dividing $p$. And in fact, $V$ is normal in $G$. Indeed if $v \in V$, then for any $g \in G$ we have $g v g^{-1} \in N$, because $V \subseteq N$ and $N$ is a normal subgroup of $G$. Hence $g v g^{-1} \in V$, because $v$ and $g v g^{-1}$ have the same order. Since $V$ is abelian, we shall write its operation additively, when considered a group on its own. We observe 
that $V$ is a left $\mathbb{Z}$-module in a natural way. In particular for a nonnegative integer $n$ and $v \in V$, we define

$$
n \times v=\underbrace{v+v+\cdots+v}_{n \text { terms }},
$$

where an empty sum is taken to be $0_{V}$. And for $n<0$, we let $n \times v=-[(-n) \times v]$.

Let $\mathbb{F}_{p}=\mathbb{Z} / p \mathbb{Z}$, the field of $p$ elements, and for an arbitrary $n \in \mathbb{Z}$, let $[n]_{p}$ denote the coset of $n$ in $\mathbb{F}_{p}$. Let $R=\mathbb{F}_{p}[H]$, the group ring of $H$ over $\mathbb{F}_{p}$. (See $[6$, p.8].) We note the $R$ is commutative, because $H$ is abelian. For $v \in V$ and $\sum_{h \in H}\left[n_{h}\right]_{p} \cdot h \in R$, define

$$
\left(\sum_{h \in H}\left[n_{h}\right]_{p} \cdot h\right) * v=\sum_{h \in H} n_{h} \times h v h^{-1} .
$$

We point out that $h v h^{-1}$ is an element of $V$, because $V$ is normal in $G$ and $H \subseteq G$. Moreover the formula of (3.2.1) is well defined, since each nontrivial element of $V$ has order $p$, and $[n]_{p}=[m]_{p}$ implies that $p$ divides $n-m$.

We claim that $V$ is a left $R$-module under the product of (3.2.1). We have $1_{R} * v=v$ for all $v \in V$, because the identity element of $H$ is also the identity of $G$. And it is easily checked that for integers $n_{h}$ and $m_{h}, h \in H$, we have

$$
\left(\sum_{h \in H}\left[n_{h}\right]_{p} \cdot h+\sum_{h \in H}\left[m_{h}\right]_{p} \cdot h\right) * v=\left(\sum_{h \in H}\left[n_{h}\right] \cdot h\right) * v+\left(\sum_{h \in H}\left[m_{h}\right] \cdot h\right) * v .
$$

For $v_{1}, v_{2} \in V$, we observe that $v_{1}+v_{2}=v_{1} v_{2}$, because the operations of $V$ and $G$ coincide on $V$. Hence for $h \in H$, we have $h\left(v_{1}+v_{2}\right) h^{-1}=h v_{1} h^{-1}+h v_{2} h^{-1}$, because each term is an element of $V$, and so we realize that

$$
\left(\sum_{h \in H}\left[n_{h}\right]_{p} \cdot h\right) *\left(v_{1}+v_{2}\right)=\left(\sum_{h \in H}\left[n_{h}\right]_{p} \cdot h\right) * v_{1}+\left(\sum_{h \in H}\left[n_{h}\right]_{p} \cdot h\right) * v_{2} .
$$


Finally, we have

$$
\left(\sum_{g \in H}\left[n_{g}\right]_{p} \cdot g\right)\left[\left(\sum_{h \in H}\left[n_{h}\right]_{p} \cdot h\right) * v\right]=\left[\left(\sum_{g \in H}\left[n_{g}\right]_{p} \cdot g\right)\left(\sum_{h \in H}\left[n_{h}\right]_{p} \cdot h\right)\right] * v
$$

because each expression is equal to $\sum_{g, h \in H}\left[\left(n_{g} n_{h}\right) \times(g h) v(g h)^{-1}\right]$. Thus our claim is established.

Now since $V$ is finite but nontrivial, there exists a minimal nontrivial submodule $W$ of $V$, obviously. In other words, $W$ is a simple $R$-module. Let $E$ be the collection of all $R$-module endomorphisms of $W$, endowed with the operations of addition and multiplication defined as follows. For arbitrary elements $\varphi, \psi \in E$ and $w \in W$, let $(\varphi+\psi)(w)=\varphi(w)+\psi(w)$ and $(\varphi \psi)(w)=\varphi(\psi(w))$. Then by Schur's Lemma, $E$ is a division ring under these operations. (Refer to [6, p.35].) Furthermore $E$ is finite, because $W \subseteq G$ and $G$ is finite. Therefore $E$ is a field, by Wedderburn's "Little" Theorem. (See [6, p.214].) Hence the multiplicative group $E^{\#}$ is cyclic (cf. [4, p.279]).

For $h \in H$, define $\varphi_{h}: W \rightarrow W$ via the rule $\varphi_{h}(w)=\left([1]_{p} \cdot h\right) * w=h w h^{-1}$, for all $w \in W$. We observe that if $x \in W$ as well, then

$$
\varphi_{h}(w+x)=h w h^{-1}+h x h^{-1}=\varphi_{h}(w)+\varphi_{h}(x)
$$

And for $\sum_{g \in H}\left[n_{g}\right]_{p} \cdot g \in R$, we have

$$
\varphi_{h}\left[\left(\sum_{g \in H}\left[n_{g}\right]_{p} \cdot g\right) * w\right]=\sum_{g \in H} n_{g} \times(h g) w(h g)^{-1}=\left(\sum_{g \in H}\left[n_{g}\right]_{p} \cdot g\right) * \varphi_{h}(w)
$$

because $R$ is commutative. Therefore $\varphi_{h} \in E$. Moreover $\varphi_{h}$ is injective, because $h w h^{-1}=h x h^{-1}$ implies $w=x$. Thus $\varphi_{h} \not \equiv 0_{W}$, and so $\varphi_{h} \in E^{\#}$. 
Define $\Phi: H \rightarrow E^{\#}$ by $\Phi(h)=\varphi_{h}$, for all $h \in H$. We observe that if $g$ is an element of $H$ as well, and $w \in W$, then

$$
\varphi_{g h}(w)=(g h) w(g h)^{-1}=g\left(h w h^{-1}\right) g^{-1}=\left(\varphi_{g} \varphi_{h}\right)(w) .
$$

Hence $\Phi(g h)=\Phi(g) \Phi(h)$. Therefore $\Phi$ is a group homomorphism, and so $\Phi(H)$ is a subgroup of $E^{\#}$. Furthermore $\Phi(H)$ must be cyclic, because $E^{\#}$ is cyclic, and a subgroup of a cyclic group is cyclic. (See [4, p.36].) But $H$ is assumed to be noncyclic. Thus $\Phi(H)$ cannot be isomorphic to $H$, and so $\Phi$ has a nontrivial kernel. In other words, there exists a nontrivial element $h \in H^{*}$ such that $\varphi_{h}$ is the identity map on $W$. Equivalently $h w h^{-1}=w$, or $h$ and $w$ are commuting elements, for all $w \in W$. Hence we have the lemma, because $W \subseteq N$ is nontrivial.

Definition 3.2.2. Let $G$ be a group, and let $p$ be a prime number. If the order of each element of $G$ is a power of $p$, then $G$ is called a p-group.

We remark that $p^{0}=1$ counts as a power of $p$ in this definition. We also note that a group $G$ is a $p$-group if and only if the order of $G$ is a power of $p$. This is a consequence of the theorems of Lagrange and Cauchy. (See [4, p.39 and p.93].)

We shall require two results concerning $p$-groups. The first is well known, and will be applied frequently; the second is more technical. We refer the reader to the literature for their arguments.

Lemma 3.2.3. If $G$ is a finite $p$-group then its center, $Z(G)$, is a nontrivial p-group. Proof. See [4, p.94]. 
Lemma 3.2.4. Let $G$ be a finite p-group. Then $G$ has a unique subgroup of order $p$ if and only if $G$ is cyclic, or a generalized quaternion group.

Proof. See [10, p.143].

Definition 3.2.5. Let $G$ be a group, and let $p$ be a prime number. If $H$ is a maximal p-subgroup of $G$, then $H$ is called a Sylow p-subgroup.

We note that for a finite group $G$ of order $p^{a} m$, where $\operatorname{gcd}(p, m)=1, H$ is a Sylow $p$-subgroup of $G$ if and only if $H$ has order $p^{a}$. Furthermore, the total number of Sylow $p$-subgroups is $\equiv(1 \bmod p)$, and any two are conjugate, thus isomorphic. (See $[4$, p.95].)

We are now prepared to give an argument for Theorem 3.1.2.

Proof. Let $N$ be a nontrivial abelian normal subgroup of $G$. Let $g_{1}$ and $g_{2}$ be arbitrary nontrivial elements of $G$, and let $i_{1}$ and $i_{2}$ be the orders of $g_{1}$ and $g_{2}$, respectively. Assume that $i_{1}=j_{1} p_{1}$ and $i_{2}=j_{2} p_{2}$, where $j_{1}$ and $j_{2}$ are positive integers, and $p_{1}$ and $p_{2}$ are prime numbers. We observe that for each $k \in\{1,2\}$, the cyclic group $\left\langle g_{k}^{j_{k}}\right\rangle$ has order $p_{k}$, and thus is a $p_{k}$-subgroup of $G$. Let $P_{k}$ be a Sylow $p_{k}$-subgroup of $G$ containing $\left\langle g_{k}^{j_{k}}\right\rangle$. Since $P_{k}$ has prime power order $p_{k}^{a_{k}}$ for some $a_{k} \geq 1, Z\left(P_{k}\right)$ is a nontrivial $p_{k}$-group, by 3.2.3. Let $z_{k} \in Z\left(P_{k}\right)^{*}$ be an element of order $p_{k}$. By hypothesis, $P_{k}$ is neither a cyclic group, nor a generalized quaternion group. Thus by 3.2.4, $P_{k}$ has more than one subgroup of order $p_{k}$. Hence there exists $x_{k} \in P_{k} \backslash\left\langle z_{k}\right\rangle$ of order $p_{k}$. 
For fixed $k \in\{1,2\}$, consider two possibilities regarding $g_{k}^{j_{k}}$. First assume that $g_{k}^{j_{k}} \in\left\langle z_{k}\right\rangle$. Then we have $x_{k} \notin\left\langle g_{k}^{j_{k}}\right\rangle$. And $x_{k}$ and $g_{k}^{j_{k}}$ are commuting elements, because $x_{k} \in P_{k}$ and $g_{k}^{j_{k}} \in Z\left(P_{k}\right)$. Therefore letting $H_{k}=\left\langle x_{k}\right\rangle \cdot\left\langle g_{k}^{j_{k}}\right\rangle$, we see that $H_{k} \simeq\left(\mathbb{Z} / p_{k} \mathbb{Z}\right) \times\left(\mathbb{Z} / p_{k} \mathbb{Z}\right)$. In particular, $H_{k}$ is a noncyclic abelian subgroup of $G$. Thus by 3.2.1, there exist nontrivial commuting elements $h_{k} \in H_{k}^{*}$ and $w_{k} \in N^{*}$. Furthermore we realize that $\left(g_{k}, g_{k}^{j_{k}}, h_{k}, w_{k}\right)$ is a commuting path in $\Delta_{G^{*}}$, because $g_{k}$ commutes with $g_{k}^{j_{k}}$, of course, and each of $g_{k}^{j_{k}}$ and $h_{k}$ is an element of the abelian group $H_{k}$.

Now assume $g_{k}^{j_{k}} \notin\left\langle z_{k}\right\rangle$. Then $g_{k}^{j_{k}}$ and $z_{k}$ generate distinct groups of order $p_{k}$. Moreover these elements commute, because $g_{k}^{j_{k}} \in P_{k}$ and $z_{k} \in Z\left(P_{k}\right)$. We therefore define $H_{k}=\left\langle g_{k}^{j_{k}}\right\rangle \cdot\left\langle z_{k}\right\rangle$, and observe that $H_{k}$ is a noncyclic abelian subgroup of $G$, once again. So as above, there exist $h_{k} \in H_{k}^{*}$ and $w_{k} \in N^{*}$ such that $\left(g_{k}, g_{k}^{j_{k}}, h_{k}, w_{k}\right)$ is a commuting path in $\Delta_{G^{*}}$.

Affixing our commuting paths for $k=1$ and $k=2$ to one another, with the $k=2$ path on the right side and written in reverse, we obtain a new path in $\Delta_{G^{*}}$, because each of $w_{1}$ and $w_{2}$ is an element of the abelian group $N$. In particular, the path is $\left(g_{1}, g_{1}^{j_{1}}, h_{1}, w_{1}, w_{2}, h_{2}, g_{2}^{j_{2}}, g_{2}\right)$. Therefore in view of the length of this path, the theorem is realized.

Before giving a proof of Corollary 3.1.3, we prove a fact from group theory.

Lemma 3.2.6. Let $G$ be a group with derived series $G=G_{0} \supseteq G_{1} \supseteq G_{2} \supseteq \cdots$. Then each $G_{i}$ is a normal subgroup of $G$. 
Proof. Let $\varphi: G \rightarrow G$ be an arbitrary homomorphism. We claim that $\varphi\left(G_{i}\right) \subseteq G_{i}$ for all $i \geq 0$. We prove this by induction on $i$. The claim is obviously true for $i=0$. So assume that it holds for some particular $i \geq 0$. Since $G_{i+1}$ is the derived subgroup of $G_{i}$, a general element of $G_{i+1}$ may be expressed as a product of commutators, $\prod_{j=1}^{k}\left[g_{j}, h_{j}\right]$, where $g_{j}, h_{j} \in G_{i}$ for $1 \leq j \leq k$. We observe that

$$
\varphi\left(\prod_{j=1}^{k}\left[g_{j}, h_{j}\right]\right)=\prod_{j=1}^{k}\left(\varphi\left(g_{j}\right) \varphi\left(h_{j}\right) \varphi\left(g_{j}\right)^{-1} \varphi\left(h_{j}\right)^{-1}\right)=\prod_{j=1}^{k}\left[\varphi\left(g_{j}\right), \varphi\left(h_{j}\right)\right] .
$$

But by assumption, we have $\varphi\left(G_{i}\right) \subseteq G_{i}$. Thus $\varphi\left(g_{j}\right), \varphi\left(h_{j}\right) \in G_{i}$ for each $j$, and so $\prod_{j=1}^{k}\left[\varphi\left(g_{j}\right), \varphi\left(h_{j}\right)\right] \in G_{i+1}$. Therefore the claim holds for $i+1$, and hence for all nonnegative integers, by induction.

Now let $x \in G$, and define $\varphi_{x}: G \rightarrow G$ via the formula $\varphi_{x}(y)=x y x^{-1}$ for all $y \in G$. Then $\varphi_{x}$ is a homomorphism. (In fact, $\varphi_{x}$ is the 'inner automorphism of $G$ induced by $x$ '. See $\left[4\right.$, pp.90-91].) Thus $\varphi_{x}\left(G_{i}\right) \subseteq G_{i}$ for all $i \geq 0$, by our above argument. The lemma follows.

We end the chapter with an argument for Corollary 3.1.3.

Proof. Let $G=G_{0} \supsetneq G_{1} \supsetneq \cdots \supsetneq G_{i-1} \supsetneq G_{i}=\left\{1_{G}\right\}$ be the derived series of the nontrivial finite solvable group $G$. We observe that the subgroup $G_{i-1}$, obviously nontrivial, is abelian, because its commutator subgroup is trivial. Furthermore, $G_{i-1}$ is a normal subgroup of $G$, by Lemma 3.2.6. Therefore since $G$ has no cyclic or generalized quaternion Sylow subgroups, by hypothesis, we realize the corollary by applying Theorem 3.1.2. 


\section{Chapter 4}

\section{Groups of Order $p^{a} q^{b}$}

\subsection{Main results}

Definition 4.1.1. Let $p$ and $q$ be distinct prime numbers, and let $G$ be a group of order $p^{a} q^{b}$, where $a$ and $b$ are positive integers. We define $M(G)$ to be the subset of all elements of $G$ whose order is divisible by both $p$ and $q$. For $r \in\{p, q\}$, we let $\mathcal{S}_{r}(G)$ denote the family of all Sylow r-subgroups of $G$. We also define $I_{r}(G)=\bigcap_{R \in \mathcal{S}_{r}(G)} R$, and $U_{r}(G)=\bigcup_{R \in \mathcal{S}_{r}(G)} R^{*}$

Informally, $M(G)$ is the collection of elements of mixed order in $G$. We observe that each $g \in G \backslash M(G)$ is either a $p$ - or a $q$-element, as implied by Lagrange's Theorem. (See $[3, \mathrm{p} .89]$.) In other words for some $r \in\{p, q\}$, the order of $g$ is a power of $r$. We also note that each such $g$ is contained in some $R \in \mathcal{S}_{r}(G)$. (See $[4$, p.94].) Hence $G^{*} \backslash M(G)=U_{p}(G) \cup U_{q}(G)$. Finally we point out that $I_{r}(G)$ is an $r$-subgroup of $G$, possibly trivial, because an arbitrary intersection of subgroups of $G$ remains a subgroup, and each $R \in \mathcal{S}_{r}(G)$ is an $r$-group. (See [3, p.61].)

We shall prove the following structure results for $\Delta_{G^{*}}$, and the group $G$ itself. 


\section{Throughout the chapter, we assume that $G$ is a group as in Definition 4.1.1,} but furthermore we take $p^{a}>q^{b}$. We remark that $p^{a}=q^{b}$ is impossible, because $\mathbb{Z}$ is a unique factorization domain. (See [3, p.287].)

Theorem 4.1.2. The commuting graph $\Delta_{G^{*}}$ is connected if and only if $M(G)$ is nonempty. Moreover if $\Delta_{G^{*}}$ is connected, then its diameter is $\leq 7$.

Theorem 4.1.3. Suppose $M(G)=\emptyset$. Then:

a. The order of $\mathcal{S}_{p}(G)$ is equal to 1 or $q^{b}$. Moreover if $\left|\mathcal{S}_{p}(G)\right|=q^{b}$, then none of the elements $P \in \mathcal{S}_{p}(G)$ is a cyclic or generalized quaternion group. Also for distinct $P, P^{\prime} \in \mathcal{S}_{p}(G), P \cap P^{\prime}=I_{p}(G)$, which is a nontrivial p-group.

b. The order of $\mathcal{S}_{q}(G)$ is equal to $\left|I_{p}(G)\right|$. Furthermore, each $Q \in \mathcal{S}_{q}(G)$ is a cyclic or generalized quaternion group, and more precisely, $Q$ is cyclic if $\left|\mathcal{S}_{p}(G)\right|>1$. In any case, for each distinct pair of elements $Q, Q^{\prime} \in \mathcal{S}_{q}(G), Q \cap Q^{\prime}$ is trivial.

c. If $\left|\mathcal{S}_{p}(G)\right|>1$, there exists a subgroup $H$ of $G$ such that $G \supsetneq H \supsetneq I_{p}(G)$ is a normal series. Furthermore, $G / H$ is a cyclic p-group, and $H / I_{p}(G)$ is a cyclic q-group.

d. The connected components of $\Delta_{G^{*}}$ are $\Delta_{U_{p}(G)}$, and $\Delta_{Q^{*}}$ for $Q \in \mathcal{S}_{q}(G)$. Moreover $\Delta_{U_{p}(G)}$ has diameter $\leq 2$ if $\left|\mathcal{S}_{p}(G)\right|=1$, but otherwise its diameter is exactly 3. The diameter of each $\Delta_{Q^{*}}$ is 1 or 2, according as $Q$ is a cyclic or generalized quaternion group, unless $q^{b}=2$, in which case the diameter is 0 . In particular the diameter of $\Delta_{Q^{*}}$ is either 0 or 1 if $\left|\mathcal{S}_{p}(G)\right|>1$. 


\subsection{The commuting graph $\Delta_{G^{*}}$ if $M(G)$ is nonempty}

Our objective here is to prove

Proposition 4.2.1. Suppose that $M(G) \neq \emptyset$. Also assume that for some $r \in\{p, q\}$, each $R \in \mathcal{S}_{r}(G)$ is a cyclic or generalized quaternion group. Then $\Delta_{G^{*}}$ is connected of diameter $\leq 6$.

We remark that, with no regard to $M(G), G$ is a solvable group by Burnside's Theorem. (See [3, p.621].) Thus if $G$ has no cyclic or generalized quaternion Sylow subgroups, then $\Delta_{G^{*}}$ is connected of diameter $\leq 7$ by Corollary 3.1.3. So as a consequence of Theorem 4.1.2, we shall realize that $M(G)$ is automatically nonempty in this situation. But here, assuming $M(G) \neq \emptyset$ and the opposite condition on the Sylow subgroups of $G$, we prove a slightly sharper bound on the diameter of $\Delta_{G^{*}}$.

We give several lemmas before the proof of 4.2 .1 . We remark that $M(G)$ is only assumed to be nonempty when this is explicitly stated. In our arguments, we shall make use of the following notation.

Definition 4.2.2. Suppose $r \in\{p, q\}$. If $g \in G$ has order ir, where $i$ is a positive integer, we define $g_{r}=g^{i}$. If $g$ is an r-element, moreover, then we let $S_{r}(g)$ be any fixed group of $\mathcal{S}_{r}(G)$ that contains $g$. For an arbitrary $R \in \mathcal{S}_{r}(G)$, we define $z_{R}$ to be any particular element of $Z(R)$ of order $r$.

We note that the element $g_{r}$ has order $r$, clearly, and thus is an $r$-element. We also point out that if $g$ is itself an $r$-element, then of course there exists a maximal 
$r$-subgroup of $G$ that contains $g$. In other words, there exists a group of $\mathcal{S}_{r}(G)$ containing $g$. Finally we remark that if $R \in \mathcal{S}_{r}(G)$, then $Z(R)$ is a nontrivial $r$-group by Lemma 3.2.3. Choosing any $z \in Z(R)^{*}$, we may let $z_{r}$ serve as $z_{R}$.

Lemma 4.2.3. Suppose $g$ and $g^{\prime}$ are elements of $M(G) \cup U_{p}(G)$. Then the distance between $g$ and $g^{\prime}$ in $\Delta_{M(G) \cup U_{p}(G)}$ is $\leq 6$.

Proof. We note that $M(G) \cup U_{p}(G)$ is the set of all elements of $G$ with order divisible by $p$. Thus $g$ and $g^{\prime}$ are as such. Let $P=S_{p}\left(g_{p}\right)$, and $P^{\prime}=S_{p}\left(g_{p}^{\prime}\right)$. Since $p^{a}>q^{b}$ by assumption, equation (2.4.1) gives

$$
\left|P P^{\prime}\right|=\frac{|P| \cdot\left|P^{\prime}\right|}{\left|P \cap P^{\prime}\right|}=\frac{\left(p^{a}\right)^{2}}{\left|P \cap P^{\prime}\right|}>\frac{|G|}{\left|P \cap P^{\prime}\right|} .
$$

But $\left|P P^{\prime}\right| \leq|G|$, because $P P^{\prime} \subseteq G$. Hence $P \cap P^{\prime}$ is nontrivial, and so there exists $x \in\left(P \cap P^{\prime}\right)^{*}$.

We observe that $z_{P}$, a central element of $P$ by definition, commutes with $g_{p}$ and $x$. Similarly, $z_{P^{\prime}}$ commutes with $g_{p}^{\prime}$ and $x$. Therefore, since $g_{p}$ and $g_{p}^{\prime}$ obviously commute with $g$ and $g^{\prime}$, respectively, we realize that $\left(g, g_{p}, z_{P}, x, z_{P^{\prime}}, g_{p}^{\prime}, g^{\prime}\right)$ is a commuting path in $\Delta_{M(G) \cup U_{p}(G)}$.

Lemma 4.2.4. Suppose that $M(G) \neq \emptyset$. Let $P \in \mathcal{S}_{p}(G)$ and $Q \in \mathcal{S}_{q}(G)$. Then there exist nontrivial commuting elements $x \in P^{*}$ and $y \in Q^{*}$.

Proof. Let $w \in M(G)$. We observe that $w_{p}$ and $w_{q}$, nontrivial $p$ - and $q$-elements respectively, commute because each is a member of the abelian group $\langle w\rangle$. Let us 
define $P^{\prime}=S_{p}\left(w_{p}\right)$, and $Q^{\prime}=S_{p}\left(w_{q}\right)$. Since all Sylow $p$-subgroups of $G$ are conjugate, there exists $u \in G$ such that $u P^{\prime} u^{-1}=P$. (Refer to [4, p.95].) Similarly, there exists $v \in G$ such that $v Q^{\prime} v^{-1}=Q$

Now, $p^{a}$ and $q^{b}$ are relatively prime integers, because $p$ and $q$ are distinct primes. Therefore $P^{\prime} \cap Q^{\prime}$, a subgroup of both $P^{\prime}$ and $Q^{\prime}$, is trivial, because its order divides $\left|P^{\prime}\right|=p^{a}$ and $\left|Q^{\prime}\right|=q^{b}$ by Lagrange's Theorem. (See [4, p.32 and p.39].) Thus by equation (2.4.1), we have $\left|P^{\prime} Q^{\prime}\right|=\left|P^{\prime}\right| \cdot\left|Q^{\prime}\right|=p^{a} q^{b}=|G|$. Hence $P^{\prime} Q^{\prime}$, which is a subset of $G$, must coincide with $G$.

Since $u, v \in G$ and $G$ is a group, we have $u^{-1} v \in G$. Select $s \in P^{\prime}$ and $t \in Q^{\prime}$ such that $s t=u^{-1} v$. Define $g=u s=v t^{-1}$. Then

$$
g P^{\prime} g^{-1}=u P^{\prime} u^{-1}=P, \quad g Q^{\prime} g^{-1}=v Q^{\prime} v^{-1}=Q
$$

because obviously $s P^{\prime} s^{-1}=P^{\prime}$ and $t^{-1} Q^{\prime} t=Q^{\prime}$. Let $x=g w_{p} g^{-1}$, and $y=g w_{q} g^{-1}$. We note that $x \in P$ and $y \in Q$, because $w_{p} \in P^{\prime}$ and $w_{q} \in Q^{\prime}$. Furthermore $x$ and $y$ are nontrivial commuting elements, because $w_{p}$ and $w_{q}$ are as such, and conjugation by $g$ induces an automorphism of $G$. (See [4, p.90].) Hence we have the lemma.

In what follows, we denote the centralizer of an element $g \in G$ by $C_{G}(g)$.

Lemma 4.2.5. Suppose that $r \in\{p, q\}$, and $R \in \mathcal{S}_{r}(G)$ is a cyclic or generalized quaternion group. Then $C_{G}\left(z_{R}\right)=\bigcup_{y \in R^{*}} C_{G}(y)$.

Proof. We observe that $\left\langle z_{R}\right\rangle$ is a subgroup of $R$ of order $r$. Thus by Lemma 3.2.4, $\left\langle z_{R}\right\rangle$ is unique with this property. Let $y \in R^{*}$, and assume that $w \in G$ commutes 
with $y$. Since $\left\langle y_{r}\right\rangle$ is a subgroup of $R$ of order $r$, we have $\left\langle z_{R}\right\rangle=\left\langle y_{r}\right\rangle$. So $z_{R}$ may be realized as a power of $y$, and hence we see that $w$ commutes with $z_{R}$. Thus $C_{G}\left(z_{R}\right) \supseteq \bigcup_{y \in R^{*}} C_{G}(y)$. On the other hand, the reverse containment is immediate, because $z_{R} \in R^{*}$.

We now proceed to the argument for Proposition 4.2.1.

Proof. Let $g$ and $g^{\prime}$ be arbitrary elements of $G^{*}$. If $g, g^{\prime} \in M(G) \cup U_{p}(G)$, then the distance between these elements in $\Delta_{G^{*}}$ is $\leq 6$ by Lemma 4.2.3.

If $g \in M(G) \cup U_{p}(G)$ and $g^{\prime} \in U_{q}(G)$, let $P=S_{p}\left(g_{p}\right)$ and $Q^{\prime}=S_{q}\left(g^{\prime}\right)$. Also let $x \in P^{*}$ and $y^{\prime} \in\left(Q^{\prime}\right)^{*}$ be commuting elements as in Lemma 4.2.4. We observe that $z_{P}$, a central element of $P$, commutes with $g_{p}$ and $x$. Similarly $z_{Q^{\prime}}$ commutes with $g^{\prime}$ and $y^{\prime}$. Thus $\left(g, g_{p}, z_{P}, x, y^{\prime}, z_{Q^{\prime}}, g^{\prime}\right)$ is a commuting path in $\Delta_{G^{*}}$.

If $g, g^{\prime} \in U_{q}(G)$, let $Q=S_{q}(g)$, and take $Q^{\prime}$ as above. Also let $P$ be an arbitrary group of $\mathcal{S}_{p}(G)$. Furthermore in view of 4.2 .4 , let $x \in P^{*}$ and $y \in Q^{*}$ be commuting elements, and likewise for $x^{\prime} \in P^{*}$ and $y^{\prime} \in\left(Q^{\prime}\right)^{*}$. On the one hand if $P$ is a cyclic or generalized quaternion group, then by Lemma $4.2 .5, z_{P}$ commutes with each of $y$ and $y^{\prime}$. Hence $\left(g, z_{Q}, y, z_{P}, y^{\prime}, z_{Q^{\prime}}, g^{\prime}\right)$ is a commuting path in $\Delta_{G^{*}}$. On the other hand if each of $Q$ and $Q^{\prime}$ is a cyclic or generalized quaternion group, then $z_{Q}$ commutes with $x$, and $z_{Q^{\prime}}$ with $x^{\prime}$, again by 4.2 .5 . Therefore $\left(g, z_{Q}, x, z_{P}, x^{\prime}, z_{Q^{\prime}}, g^{\prime}\right)$ is a path in $\Delta_{G^{*}}$.

We now see that in any case, the distance between $g$ and $g^{\prime}$ in $\Delta_{G^{*}}$ is $\leq 6$. Thus we have the proposition. 


\subsection{The structure of $G$ and $\Delta_{G^{*}}$ if $M(G)$ is empty}

In this section, we shall ultimately prove Theorem 4.1.3. In what follows, we assume that $M(G)$ empty.

Lemma 4.3.1. Suppose that $x$ and $y$ are nontrivial $p$ - and q-elements of $G$, respectively. Then $C_{G}(x)$ is a p-group, and $C_{G}(y)$ is a q-group. Furthermore, $x$ and $y$ do not commute.

Proof. Assume that $C_{G}(x)$ contains a nontrivial $q$-element $w$. Then the order of $x w$ is the least common multiple of those of $x$ and $w$, because $x$ and $w$ commute. (Refer to $[3$, p.156].) However the order of $x$ is obviously divisible by $p$, while that of $y$ is divisible by $q$. Therefore the least common multiple of the pair of orders is divisible by $p$ and $q$. Hence $x w$ has mixed order, contradicting that $M(G)$ is empty. It follows that $C_{G}(x)$ is a $p$-group, and by analogy, $C_{G}(y)$ is a $q$-group. In particular, we observe that $x \notin C_{G}(y)$.

Lemma 4.3.2. Suppose that $r_{1}=p$ and $r_{2}=q$, or vice versa. Assume that $x$ is a nontrivial $r_{1}$-element of $G$, and $R_{2}$ is an $r_{2}$-subgroup of $G$. Then the function $y \mapsto y x y^{-1}$, mapping $R_{2}$ to $G$, is injective. Furthermore, if $R_{1}$ is a nontrivial normal $r_{1}$-subgroup of $G$, then $\left|R_{2}\right| \leq\left|R_{1}^{*}\right|$.

Proof. Suppose $y$ and $y^{\prime}$ are distinct elements of $R_{2}$ such that $y x y^{-1}=\left(y^{\prime}\right) x\left(y^{\prime}\right)^{-1}$. Then $\left(y^{\prime}\right)^{-1} y$ commutes with $x$. But $\left(y^{\prime}\right)^{-1} y \in R_{2}$, because $y, y^{\prime} \in R_{2}$ and $R_{2}$ is a group. Thus $\left(y^{\prime}\right)^{-1} y$ is an $r_{2}$-element of $G$. Moreover $\left(y^{\prime}\right)^{-1} y$ is nontrivial, because 
$y$ and $y^{\prime}$ are distinct. Hence we have contradicted Lemma 4.3.1, and so we conclude that $y \mapsto y x y^{-1}$ is injective.

Now assuming that $x \in R_{1}$, we have $y x y^{-1} \in R_{1}$ for all $y \in R_{2}$, because $R_{1}$ is normal in $G$. Moreover $y x y^{-1}$ is a nontrivial element, since $x$ is nontrivial. Therefore $\left|R_{2}\right| \leq\left|R_{1}^{*}\right|$, because $y \mapsto y x y^{-1}$ is injective.

Lemma 4.3.3. There exists a nontrivial abelian normal p-subgroup $N$ of $G$.

Proof. As we have previously noted, $G$ is solvable by Burnside's Theorem. (Again see [3, p.621].) Let $G=G_{0} \supsetneq G_{1} \supsetneq \cdots \supsetneq G_{i-1} \supsetneq G_{i}=\left\{1_{G}\right\}$ be the derived series of $G$, and let $N=G_{i-1}$. As in the proof of Corollary 3.1.3, $N$ is an abelian normal subgroup of $G$. We claim that $N$ is a $p$-group, moreover. If the order of $N$ is divisible by both $p$ and $q$, then $N$ contains nontrivial $p$ - and $q$-elements $x$ and $y$, by Cauchy's Theorem. (See [4, p.93].) Moreover $x$ and $y$ commute, because $N$ is abelian. However, this contradicts Lemma 4.3.1.

Now assume that $N$ is a $q$-group, and let $P$ be an arbitrary member of $\mathcal{S}_{p}(G)$. Then by Lemma 4.3.2, we have $|P| \leq\left|N^{*}\right|$, because $N$ is also normal in $G$. However $N$, like any $q$-subgroup of $G$, has order $\leq q^{b}$. (Refer to [4, pp.94-95].) Therefore $|N|<p^{a}$, because $q^{b}<p^{a}$ by assumption. But $|P|=p^{a}$; so we have a contradiction once again. Hence we have realized our claim, that $N$ is a $p$-group.

Lemma 4.3.4. Suppose that $r \in\{p, q\}$, and $R$ is an $r$-group. Furthermore assume that $R$ is neither a cyclic nor a generalized quaternion group. Then $R$ contains a noncyclic abelian subgroup $H$. 
Proof. We observe that $\left\langle z_{R}\right\rangle$ is a subgroup of $R$ of order $r$, by the definition of $z_{R}$. In view of 3.2.4, let $\langle y\rangle$ be another such subgroup, distinct from $\left\langle z_{R}\right\rangle$. Since $z_{R}$ is central in $R, y$ and $z_{R}$ commute. Therefore $H$, which we define to be $\langle y\rangle \cdot\left\langle z_{R}\right\rangle$, is an abelian subgroup of $R$. (See [3, p.62].) Now $\langle y\rangle \cap\left\langle z_{R}\right\rangle$ is a proper subgroup of each of $\langle y\rangle$ and $\left\langle z_{R}\right\rangle$, because an intersection of groups remains a group, and $\langle y\rangle \neq\left\langle z_{R}\right\rangle$. (See [3, p.61].) Therefore the order of $\langle y\rangle \cap\left\langle z_{R}\right\rangle$ is a proper divisor of $r$, the order of $\langle y\rangle$ and $\left\langle z_{R}\right\rangle$ alike, by Lagrange's Theorem. Hence $\langle y\rangle \cap\left\langle z_{R}\right\rangle$ is trivial, because $r$ is a prime. So equation (2.4.1) implies $|H|=|\langle y\rangle| \cdot\left|\left\langle z_{R}\right\rangle\right|=r^{2}$. But for $i, j \in \mathbb{Z}$, $\left(y^{i} z_{R}^{j}\right)^{r}=\left(y^{r}\right)^{i}\left(z_{R}^{r}\right)^{j}=1_{R}$, since $y$ and $z$ commute. Thus $H$ is noncyclic.

Lemma 4.3.5. Each $Q \in \mathcal{S}_{q}(G)$ is either a cyclic or generalized quaternion group. Accordingly $\Delta_{Q^{*}}$ is a connected component of $\Delta_{G^{*}}$ of diameter 1 or 2 , unless $q^{b}=2$, in which case the diameter is 0 .

Proof. Assume that $Q \in \mathcal{S}_{q}(G)$ is neither a cyclic nor a generalized quaternion group. Then by Lemma 4.3.4, $Q$ contains a noncyclic abelian subgroup $H$. Letting $N$ be as in Lemma 4.3.3, we observe that there exist nontrivial commuting elements $x \in N^{*}$ and $y \in H^{*}$, by Lemma 3.2.1. But $N$ is a $p$-group, and $H$ is a $q$-group; therefore $x$ is a $p$-element and $y$ is a $q$-element. Thus we have contradicted Lemma 4.3.1. Hence we conclude that $Q$ is a cyclic or generalized quaternion group.

On the one hand if $Q$ is cyclic, then $Q$ is abelian, and hence $\Delta_{Q^{*}}$ is connected of diameter 0 or 1 according as $q^{b}=2$ or $q^{b}>2$. On the other hand if $Q$ is a generalized quaternion group, then $Q$ is non-abelian; for example $y x=x^{-1} y=\left(x^{2^{n-1}-1}\right) y \neq x y$, 
because $2^{n-1}>2$ for $n \geq 3$. (See Definition 3.1.1.) Hence $\Delta_{Q^{*}}$ has diameter $>1$. But given any elements $y, y^{\prime} \in Q^{*}$, we recall that $z_{Q}$ is defined to be a nontrivial central element of $Q$, and we observe that $\left(y, z_{Q}, y^{\prime}\right)$ is a commuting path in $\Delta_{Q^{*}}$. Therefore $\Delta_{Q^{*}}$ is connected of diameter 2 .

Now suppose that $\Delta_{Q^{*}}$ is not a connected component of $\Delta_{G^{*}}$. Then there exist commuting elements $u \in Q^{*}$ and $t \in G \backslash Q$. By Lemma 4.2.5, $z_{Q}$ commutes with $t$ as well. Therefore $C_{G}\left(z_{Q}\right) \supsetneq Q$, because $z_{Q}$ is a central element of $Q$. Hence $C_{G}\left(z_{Q}\right)$ is not a $q$-group, since $Q$ is a maximal $q$-subgroup of $G$. So once again, we have contradicted Lemma 4.3.1. Therefore, the proof is complete.

Lemma 4.3.6. If $\left|\mathcal{S}_{p}(G)\right|=1$, then $\left|\mathcal{S}_{q}(G)\right|=p^{a}$. Moreover, any distinct pair of Sylow q-subgroups of $G$ has a trivial intersection.

Proof. By Sylow theory, the number of Sylow $q$-subgroups of $G$ is a divisor of $p^{a}$. (Refer to [3, p.141].) Suppose $\left|\mathcal{S}_{q}(G)\right|=p^{e}$, where $e$ is a positive integer $\leq a$. Then we have $\left|U_{q}(G)\right| \leq p^{e}\left(q^{b}-1\right)$, with equality if and only if any distinct pair of elements of $\mathcal{S}_{q}(G)$ has a trivial intersection. But $\left|U_{p}(G)\right|=p^{a}-1$, because $\left|\mathcal{S}_{p}(G)\right|=1$ by hypothesis. Therefore,

$$
|G| \leq p^{a}+p^{e}\left(q^{b}-1\right)=p^{e}\left(p^{a-e}+q^{b}-1\right)
$$

because $M(G)$ is assumed to be empty. Hence $p^{a-e} q^{b} \leq p^{a-e}+q^{b}-1$, which implies that $p^{a-e}\left(q^{b}-1\right) \leq q^{b}-1$. Thus we have $a=e$. Furthermore, $\left|U_{q}(G)\right|=p^{e}\left(q^{b}-1\right)$, because the inequality of (4.3.1) is saturated. Therefore, each distinct pair of Sylow 
$q$-subgroups of $G$ has a trivial intersection.

Lemma 4.3.7. Assume that $P \in \mathcal{S}_{p}(G)$ is a cyclic or generalized quaternion group. Then $P$ is normal in $G$, and in fact $P$ is cyclic. Moreover each $Q \in \mathcal{S}_{q}(G)$ is cyclic.

Proof. In the proof of Lemma 4.3.5, we showed that if $Q \in \mathcal{S}_{q}(G)$ is a cyclic or generalized quaternion group, then $\Delta_{Q^{*}}$ is a connected component of $\Delta_{G^{*}}$. The same argument applies to $P$ here. Supposing that $P^{\prime} \in \mathcal{S}_{p}(G) \backslash\{P\}$, let $g \in P^{*}$, and $g^{\prime} \in P^{\prime} \backslash P$. Then by Lemma 4.2.3, $g$ and $g^{\prime}$ are in the same connected component of $\Delta_{G^{*}}$. However, this contradicts that $\Delta_{P^{*}}$ is itself a component of $\Delta_{G^{*}}$. Therefore $\mathcal{S}_{p}(G)=\{P\}$, or in other words, $P$ is a normal subgroup of $G$. (See [4, p.95].)

We observe that $\left\langle z_{P}\right\rangle$ is a subgroup of $P$ of order $p$, by the definition of $z_{P}$. Moreover by Lemma 3.2.4, $\left\langle z_{P}\right\rangle$ is unique with this property. But we observe that for $x \in G, x\left\langle z_{P}\right\rangle x^{-1}$ is a subgroup of $P$ of order $p$, because $P$ is normal in $G$ and conjugation by a fixed element induces an automorphism of $G$. (See $[4$, p.90].) Therefore $x\left\langle z_{P}\right\rangle x^{-1}=\left\langle z_{P}\right\rangle$, and so $\left\langle z_{P}\right\rangle$ is a normal subgroup of $G$. Thus for a fixed $Q \in \mathcal{S}_{q}(G)$, we have $|Q| \leq p-1$ by Lemma 4.3.2. Since $Q$ is nontrivial, it follows that $p \neq 2$. Therefore $P$ is not a generalized quaternion group, and so $P$ is cyclic in view of our hypothesis. Let us assume that $P=\langle x\rangle$, where $x \in P$ has order $p^{a}$.

Now let $y \in Q$. Since $P$ is normal in $G$, we have $y x y^{-1} \in P$. And $y x y^{-1}$ is nontrivial, because $x$ is nontrivial. Thus there exists $e(y) \in\left\{1,2, \ldots, p^{a}-1\right\}$ such that $y x y^{-1}=x^{e(y)}$. Furthermore, the map $y \mapsto e(y)$ is injective on $Q$, because this is 
true of $y \mapsto y x y^{-1}$ by Lemma 4.3.2. Letting $y^{\prime}$ be an element of $Q$ as well, we observe

$$
x^{e\left(y y^{\prime}\right)}=\left(y y^{\prime}\right) x\left(y y^{\prime}\right)^{-1}=y x^{e\left(y^{\prime}\right)} y^{-1}=\prod_{i=1}^{e\left(y^{\prime}\right)} y x y^{-1}=\prod_{i=1}^{e\left(y^{\prime}\right)} x^{e(y)}=x^{e(y) e\left(y^{\prime}\right)} .
$$

Likewise $x^{e\left(y^{\prime} y\right)}=x^{e(y) e\left(y^{\prime}\right)}$; hence $x^{e\left(y y^{\prime}\right)}=x^{e\left(y^{\prime} y\right)}$. And we observe that if $i$ and $j$ are distinct elements of $\left\{1,2, \ldots, p^{a}-1\right\}$, then $x^{i}$ and $x^{j}$ are distinct; for otherwise, assuming $i<j$, we must conclude that $x$ has order $\leq j-i$. Thus $e\left(y y^{\prime}\right)=e\left(y^{\prime} y\right)$, and so $y y^{\prime}=y^{\prime} y$ because $e$ is injective. It follows that $Q$ is abelian, and in particular, $Q$ is not a generalized quaternion group. (See the proof of 4.3.5.) Therefore $Q$ is cyclic, in view of the result of Lemma 4.3.5.

We now give arguments for Theorem 4.1.3, organized into two separate proofs. Our first deals with the structure of $G$, the second with $\Delta_{G^{*}}$. We begin with assertions a-c of 4.1.3, regarding the group $G$.

Proof. First assume that $\left|\mathcal{S}_{p}(G)\right|=1$. Then by Lemma 4.3.6, $\left|\mathcal{S}_{q}(G)\right|=p^{a}=\left|I_{p}(G)\right|$, and each distinct pair of Sylow $q$-subgroups of $G$ has a trivial intersection. Furthermore, any element $Q \in \mathcal{S}_{q}(G)$ is a cyclic or generalized quaternion group, by Lemma 4.3.5.

Now assume that $\left|\mathcal{S}_{p}(G)\right|>1$. It is well known that $I_{p}(G)$, itself a normal $p$ subgroup of $G$, contains every such subgroup of $G$. (See [3, p.149].) Assume that $\left|I_{p}(G)\right|=p^{e}$, where $e$ is a nonnegative integer, and let us define $\bar{G}=G / I_{p}(G)$. Then $\bar{G}$ is a group of order $p^{a-e} q^{b}$ (cf. [4, p.42]), and we observe that $a>e$ because $\left|\mathcal{S}_{p}(G)\right|>1$. Therefore $\bar{G}$ has order divisible by $p$ and $q$. But we claim that $\bar{G}$ has no 
elements of mixed order. Suppose that $x \in G$ has order $i$, and note that $i$ is a power of $p$ or $q$, because $M(G)=\emptyset$. Since $x^{i} \in I_{p}(G)$, there exists a minimum positive integer $j$ such that $x^{j} \in I_{p}(G)$. Let $k$ be the order of $x^{j}$. Then obviously we have $i=j k$; hence $j$ is a power of $p$ or $q$. But $j$ is also the order of the $\operatorname{coset} x \cdot I_{p}(G) \in \bar{G}$. Thus we have the claim.

Suppose $p^{a-e}>q^{b}$. Then by Lemma $4.3 .3, \bar{G}$ has a nontrivial normal $p$-subgroup $\bar{N}$. Moreover, $\bar{N}$ clearly takes the form $N / I_{p}(G)$, where $N$ is a normal subgroup of $G$ that strictly contains $I_{p}(G)$. And we observe that $N$ is itself a $p$-group, because $\bar{N}$ and $I_{p}(G)$ are as such, and $|N|=\left|I_{p}(G)\right| \cdot|\bar{N}|$. (Again refer to [4, p.42]). But this contradicts the maximality of $I_{p}(G)$, as specified above. Therefore $p^{a-e}<q^{b}$, since equality is impossible, and so $e>0$ because $p^{a}>q^{b}$. Hence $I_{p}(G)$ is nontrivial.

Fix $Q \in \mathcal{S}_{q}(G)$, and define $H=I_{p}(G) \cdot Q$. Since $I_{p}(G)$ is a normal subgroup of $G, H$ is a subgroup of $G$ containing both $I_{p}(G)$ and $Q$. (See [3, p.94].) We observe that the subgroup $I_{p}(G) \cap Q$, of $I_{p}(G)$ and $Q$ alike, is trivial by Lagrange's Theorem, because $\left|I_{p}(G)\right|=p^{e}$ and $|Q|=q^{b}$ are relatively prime. Therefore $H$ has order $p^{e} q^{b}$, by equation (2.4.1). And we have $p^{e}>q^{b}$, by Lemma 4.3 .2 with $R_{1}=I_{p}(G)$ and $R_{2}=Q$

Now $H / I_{p}(G)$ is isomorphic to $Q$, by the diamond isomorphism theorem, because $I_{p}(G) \cap Q$ is trivial. (See [3, p.97].) In particular, $\left|H / I_{p}(G)\right|=|Q|=q^{b}$. Thus we see that $H / I_{p}(G)$ is a Sylow $q$-subgroup of $\bar{G}$, because $|\bar{G}|=p^{a-e} q^{b}$. But $Q$, and hence $H / I_{p}(G)$, is a cyclic or generalized quaternion group by Lemma 4.3.5. So furthermore 
by Lemma 4.3.7, with the roles of $p$ and $q$ reversed because $p^{a-e}<q^{b}, H / I_{p}(G)$ is cyclic and normal in $\bar{G}$; thus $Q$ is cyclic. Moreover each Sylow $p$-subgroup of $\bar{G}$ is cyclic.

Since $H / I_{p}(G)$ is normal in $\bar{G}, H$ is obviously a normal subgroup of $G$. Therefore since $H$ contains $Q$, and all Sylow $q$-subgroups of $G$ are conjugate (cf. [4, p.95]), $H$ contains each Sylow $q$-subgroup of $G$. On the other hand we observe that $I_{p}(G)$ is a Sylow $p$-subgroup of $H$, because $H / I_{p}(G)$ is a $q$-group. Furthermore $I_{p}(G)$ is normal in $H$, since normal in $G$. Thus $\mathcal{S}_{p}(H)=\left\{I_{p}(G)\right\}$. Therefore by Lemma 4.3.6, the number of Sylow $q$-subgroups of $H$, and hence of $G$, is $p^{e}=\left|I_{p}(G)\right|$. Also, each distinct pair of elements of $\mathcal{S}_{q}(G)$ has a trivial intersection.

The fact that $\left|\mathcal{S}_{q}(\bar{G})\right|=1$ also implies $\left|\mathcal{S}_{p}(\bar{G})\right|=q^{b}$, in view of Lemma 4.3.6 once again. Furthermore each distinct pair of Sylow $p$-subgroups of $\bar{G}$ has a trivial intersection. But obviously $\mathcal{S}_{p}(\bar{G})=\left\{P / I_{p}(G) \mid P \in \mathcal{S}_{p}(G)\right\}$. Thus $\left|\mathcal{S}_{p}(G)\right|=q^{b}$ as well, but the intersection of each distinct pair of elements of $\mathcal{S}_{p}(G)$ is $I_{p}(G)$. In particular we point out that $\left|\mathcal{S}_{p}(G)\right|>1$, so no member of $\mathcal{S}_{p}(G)$ is a cyclic or generalized quaternion group, by Lemma 4.3.7.

For a fixed $P \in \mathcal{S}_{p}(G)$, we observe that $P / I_{p}(G)$ is a cyclic group, because $P / I_{p}(G) \in \mathcal{S}_{p}(\bar{G})$. We claim that $\varphi: x \cdot I_{p}(G) \mapsto x \cdot H$ is an isomorphism of $P / I_{p}(G)$ with $G / H$. Since $I_{p}(G)$ is a subgroup of $H, \varphi$ is well defined. And $\varphi$ is multiplicative, by the definition of coset multiplication in a quotient group. Suppose that $\varphi\left(x \cdot I_{p}(G)\right)=\varphi\left(x^{\prime} \cdot I_{p}(G)\right)$ for some $x, x^{\prime} \in P$. Then we have $\left(x^{\prime}\right)^{-1} x \in H$, 
and of course $\left(x^{\prime}\right)^{-1} x \in P$. But $H \cap P=I_{p}(G)$, because $I_{p}(G)$ is a Sylow $p$-subgroup of $H$. Thus $x \cdot I_{p}(G)=x^{\prime} \cdot I_{p}(G)$, so it follows that $\varphi$ is injective. Therefore we have the claim, because $\left|P / I_{p}(G)\right|=|G / H|=p^{a-e}$. We conclude that $G / H$ is a cyclic p-group.

We finally give our argument for Theorem 4.1.3, part $\mathrm{d}$, concerning $\Delta_{G^{*}}$.

Proof. Suppose $\mathcal{S}_{p}(G)=\{P\}$. Then $\Delta_{U_{p}(G)}=\Delta_{P^{*}}$, which has diameter $\leq 2$ because $P$ has a nontrivial center by Lemma 3.2.3. In particular, given any pair of elements $x, x^{\prime} \in P^{*},\left(x, z_{P}, x^{\prime}\right)$ is a commuting path in $\Delta_{P^{*}}$. On the other hand for each $Q \in \mathcal{S}_{q}(G), \Delta_{Q^{*}}$ is connected component of $\Delta_{G^{*}}$ by Lemma 4.3 .5 ; hence $\Delta_{P^{*}}$ must be a connected component as well. Moreover each $Q$ is a cyclic or generalized quaternion group, with the diameter of $\Delta_{Q^{*}}$ being 1 or 2 accordingly, unless $q^{b}=2$, where we have diameter 0 .

Now assume that $\left|\mathcal{S}_{p}(G)\right|>1$. Then each $Q \in \mathcal{S}_{q}(G)$ is cyclic, as shown in the proof above; thus $\Delta_{Q^{*}}$, which remains a connected component of $\Delta_{G^{*}}$, has diameter 0 or 1 . Regarding $\Delta_{U_{p}(G)}$, let $P_{1}$ and $P_{2}$ be distinct elements of $\mathcal{S}_{p}(G)$. For $i \in\{1,2\}$, select $x_{i} \in P_{i} \backslash I_{p}(G)$, and suppose that there exists a $p$-element $y$ commuting with each $x_{i}$. Then by $4.3 .1, C_{G}(y)$ is a $p$-group; hence there exists $P \in \mathcal{S}_{p}(G)$ containing $C_{G}(y)$. In particular, $P$ contains $x_{1}$ and $x_{2}$. Thus $P \cap P_{1} \nsubseteq I_{p}(G)$, and $P \cap P_{2} \nsubseteq I_{p}(G)$. It follows that $P=P_{1}$ and $P=P_{2}$, since each distinct pair of Sylow $p$-subgroups of $G$ has intersection $I_{p}(G)$, as established in the earlier proof. But this contradicts that $P_{1} \neq P_{2}$. Therefore $d_{U_{p}(G)}\left(x_{1}, x_{2}\right) \geq 3$, and so the diameter of $\Delta_{U_{p}(G)}$ is $\geq 3$. 
For the reverse inequality, let $N$ be a nontrivial abelian normal $p$-subgroup of $G$, as in Lemma 4.3.3. Also let $x_{1}$ and $x_{2}$ be arbitrary nontrivial $p$-elements of $G$, and let $P_{1}, P_{2} \in \mathcal{S}_{p}(G)$ contain $x_{1}$ and $x_{2}$ respectively. Since $N$ is normal in $G$, and any conjugate of a nontrivial element remains nontrivial, each $P_{i}$ acts on $N^{*}$ by conjugation. We point out that the index of any subgroup of $P_{i}$ is a power of $p$, because $P_{i}$ is a $p$-group. So likewise, the number of elements of any orbit under the action of $P_{i}$ is a power of $p$. (See $\left[4\right.$, p.89].) But $\left|N^{*}\right|=|N|-1$ is not divisible by $p$, because $N$ is a $p$-group. Hence the action of $P_{i}$ yields a singleton orbit, say $\left\{z_{i}\right\}$. Therefore $z_{i}$ commutes with each element of $P_{i}$, and in particular with $x_{i}$. We observe that $z_{1}$ and $z_{2}$ themselves commute, because $N$ is abelian. Moreover $z_{1}$ and $z_{2}$, as members of $N^{*}$, are nontrivial $p$-elements. Thus $\left(x_{1}, z_{1}, z_{2}, x_{2}\right)$ is a commuting path in $\Delta_{U_{p}(G)}$. We conclude that $\Delta_{U_{p}(G)}$ has diameter $\leq 3$, and furthermore $\Delta_{U_{p}(G)}$ is a connected component of $\Delta_{G^{*}}$, because each $\Delta_{Q^{*}}$ is as such.

\subsection{Examples}

In this section we exhibit a pair of constructions, each yielding a group of order $p^{a} q^{b}$ with no elements of mixed order.

Example 4.4.1. Let $p$ be an odd prime, let $q$ be any prime divisor of $p-1$, and let $n$ be an arbitrary element of $\{2,3, \ldots, q\}$. Define $\mathbb{F}_{p}=\mathbb{Z} / p \mathbb{Z}$, the field of $p$ elements, 
and define

$$
P=\left\{\left(a_{i j}\right) \in G L_{n}\left(\mathbb{F}_{p}\right) \mid a_{i j}=0 \text { for } i<j \text {, and } a_{i i}=1 \text { for } 1 \leq i \leq n\right\}
$$

Then $P$ is obviously a group, and we have $|P|=p^{s}$, where $s=1+2+\cdots+(n-1)$. Hence, $P$ is a $p$-group.

By Cauchy's Theorem, the multiplicative group $F_{p}^{\#}$, of order $p-1$, has an element $\zeta$ of order $q$. Let

$$
Q=\left\langle\operatorname{diag}\left(1, \zeta, \zeta^{2}, \ldots, \zeta^{n-1}\right)\right\rangle \subseteq P
$$

We observe that $Q$ acts on $P$ by conjugation, and thus we may form the semidirect product $P \rtimes Q$. Let $G$ denote this group, of order $p^{s} q$.

Suppose that $G$ contains an element $\left(C_{1}, D_{1}\right)$ of order divisible by $p$ and $q$. Taking appropriate powers of $\left(C_{1}, D_{1}\right)$, we obtain a commuting pair of elements $\left(C_{2}, D_{2}\right)$ and $\left(C_{3}, D_{3}\right)$ having respective orders $p$ and $q$. Define

$$
\tilde{P}=\left\{\left(A, \operatorname{id}_{n}\right) \mid A \in P\right\}, \quad \tilde{Q}=\left\{\left(\operatorname{id}_{n}, B\right) \mid B \in Q\right\}
$$

Obviously $\tilde{P}$ and $\tilde{Q}$ are subgroups of $G$, and in fact $\tilde{P} \in \mathcal{S}_{p}(G)$ and $\tilde{Q} \in \mathcal{S}_{q}(G)$, in view of their orders. Furthermore $\tilde{P}$ is normal in $G$, clearly; therefore $\left(C_{2}, D_{2}\right) \in \tilde{P}$. And since all Sylow $q$-subgroups of $G$ are conjugate, we may take $\left(C_{3}, D_{3}\right) \in \tilde{Q}$. Assume that $\left(C_{2}, D_{2}\right)=\left(A, \mathrm{id}_{n}\right)$ and $\left(C_{3}, D_{3}\right)=\left(\operatorname{id}_{n}, B\right)$, where $A \in P$ and $B \in Q$. We note that $A$ and $B$ have orders $p$ and $q$, respectively, so in particular these elements are 
nontrivial. We observe

$$
\begin{aligned}
& \left(C_{2}, D_{2}\right) \cdot\left(C_{3}, D_{3}\right)=(A, B) \\
& \left(C_{3}, D_{3}\right) \cdot\left(C_{2}, D_{2}\right)=\left(B A B^{-1}, B\right) .
\end{aligned}
$$

Therefore $A=B A B^{-1}$, because $\left(C_{2}, D_{2}\right)$ and $\left(C_{3}, D_{3}\right)$ are commuting elements.

Since $B$ is a nontrivial element of $Q$, we have $B=\operatorname{diag}\left(1, \zeta^{e}, \zeta^{2 e}, \ldots, \zeta^{(n-1) e}\right)$ for some $e \in\{1,2, \ldots, q-1\}$. Thus

$$
\left(B A B^{-1}\right)_{i j}=\zeta^{(i-1) e} \cdot a_{i j} \cdot \zeta^{-(j-1) e}=\zeta^{(i-j) e} \cdot a_{i j} .
$$

Since $A$ is nontrivial, there exist $i, j \in\{1,2, \ldots, n\}, i>j$, such that $a_{i j} \neq 0$. Hence $\zeta^{(i-j) e}=1$, because $A=B A B^{-1}$. It follows that $q$ divides $(i-j) e$, since $\zeta$ has order $q$. Thus $q$ divides $i-j$ or $e$, because $q$ is a prime. But $q$ does not divide $e$, clearly, and furthermore $q$ does not divide $i-j$, because $1 \leq i-j<n \leq q$. Therefore we have a contradiction, and so $G$ has no elements of mixed order.

We remark that the above example generalizes to the case where $q$ is replaced by $q^{b}$, provided that $q^{b}$ is a divisor of $p-1$ and $q$ remains $>n$. However the existence of an element $\zeta \in \mathbb{F}_{p}^{\#}$ of order $q^{b}$ is not implied by Cauchy's Theorem in this situation. We may instead cite the fact that $\mathbb{F}_{p}^{\#}$ is cyclic, as is well known.

Example 4.4.2. For a positive integer $n$, let $Q$ denote the cyclic or generalized quaternion group of order $2^{n}$. (If $n \in\{1,2\}$, we take $Q$ to be cyclic.) Referring to 3.2.3 and 3.2.4, let $z_{Q}$ denote the unique element of $Q$ of order 2 , and note that 
$z_{Q} \in Z(Q)$. Let $p$ be an odd prime, and let $R$ denote the group ring $\mathbb{F}_{p}[Q]$, of order $p^{2^{n}}$. Define

$$
V=\left\{s \in R \mid s+\left(1_{\mathbb{F}_{p}} \cdot z_{Q}\right) s=0_{R}\right\}
$$

We observe that $1_{\mathbb{F}_{p}} \cdot 1_{Q}+\left(-1_{\mathbb{F}_{p}}\right) \cdot z_{Q} \in V$, because $z_{Q}$ has order 2 . Moreover, $V$ is obviously closed under addition; therefore $V$ is a nontrivial additive $p$-group, say of order $p^{m}$. We also observe that $g \in Q$ and $s \in V$ implies $\left(1_{\mathbb{F}_{p}} \cdot g\right) s \in V$, because $g$ commutes with $z_{Q}$. Thus $Q$ acts on $V$.

Let $G$ denote the semidirect product $V \rtimes Q$, of order $p^{m} 2^{n}$. Suppose $G$ contains an element of mixed order, divisible by $p$ and 2. Then arguing as in the previous example, we realize that there exists $s \in V$ of order $p$ and $g \in Q$ of order 2 such that $\left(s, 1_{Q}\right)$ and $\left(0_{V}, g\right)$ are commuting elements of $G$. Furthermore we have $g=z_{Q}$, because of the uniqueness of $z_{Q}$. Hence, taking the product of the commuting elements in each possible order, we find that

$$
(s, g)=\left(\left(1_{\mathbb{F}_{p}} \cdot z_{Q}\right) s, g\right) .
$$

Thus $s=\left(1_{\mathbb{F}_{p}} \cdot z_{Q}\right) s$. However $s=\left(\left(-1_{\mathbb{F}_{p}}\right) \cdot z_{Q}\right) s$, because $s \in V$. Therefore since $s$ is nontrivial, we have $1_{\mathbb{F}_{p}}=-1_{\mathbb{F}_{p}}$. But this is a contradiction, because $p$ is odd. Hence $G$ has no elements of mixed order.

\subsection{A condition for connectivity of $\Delta_{G^{*}}$}

Here we formalize a brief argument for Theorem 4.1.2. 
Proof. As we have noted more than once, $G$ is a solvable group by Burnside's Theorem. (See [3, p.621].) Therefore if $G$ has no cyclic or generalized quaternion Sylow subgroups, then $\Delta_{G^{*}}$ is connected of diameter $\leq 7$ by Corollary 3.1.3. On the other hand if $G$ contains a cyclic or generalized quaternion Sylow subgroup, and $M(G)$ is nonempty, then in fact $\Delta_{G^{*}}$ has diameter $\leq 6$ by Proposition 4.2 .1 . But by Theorem 4.1.3, $\Delta_{G^{*}}$ is disconnected if $M(G)$ is empty.

We point out that in the proof we might have said "if $G$ has no cyclic or generalized quaternion Sylow subgroups, and $M(G)$ is nonempty, then...". However this was logically unnecessary, because of 3.1.3. Thus, as we have mentioned previously, $M(G)$ is automatically nonempty if $G$ has no cyclic or generalized quaternion Sylow subgroups. 


\section{Bibliography}

[1] C. Bates et al., Commuting involution graphs for symmetric groups, J. Algebra 266 (2003), 133-153.

[2] D. Bundy, The connectivity of commuting graphs, J. Combinatorial Theory 113 (2006), 995-1007.

[3] D. Dummit and R. Foote, Abstract Algebra, Prentice-Hall, Englewood Cliffs, NJ, 1991.

[4] T. Hungerford, Algebra, Graduate Texts in Mathematics 73, Springer-Verlag, New York, NY, 1974.

[5] N. Jacobson, Basic Algebra I, Second Edition, W.H. Freeman and Company, New York, NY, 1985

[6] T. Lam, A First Course in Noncommutative Rings, Graduate Texts in Mathematics 131, Springer-Verlag, New York, NY, 1991. 
[7] A. Potapchik and A. Rapinchuk, Normal subgroups of $S L_{1, D}$ and the classification of finite simple groups, Proc. Indian Acad. Sci. 106 (1996), 329-368.

[8] A. Rapinchuk and Y. Segev, Valuation-like maps and the congruence subgroup property, Invent. math. 144 (2001), 571-607.

[9] A. Rapinchuk, Y. Segev, and G. Seitz, Finite quotients of the multiplicative group of a finite dimensional division algebra are solvable, J. Amer. Math. Soc. 15 (2002), 929-978.

[10] D. Robinson, A Course in the Theory of Groups, Second Edition, Graduate Texts in Mathematics 80, Springer-Verlag, New York, NY, 1996.

[11] Y. Segev, On finite homomorphic images of the multiplicative group of a division algebra, Ann. of Math. 149 (1999), 219-251.

[12] Y. Segev and G. Seitz, Anisotropic groups of type $A_{n}$ and the commuting graph of finite simple groups, Pacific J. Math. 202 (2002), 125-225. 